\title{
Neutrosophic Semiopen Hypersoft Sets with an Application to MAGDM under the COVID-19 Scenario
}

\author{
D. Ajay, ${ }^{1}$ J. Joseline Charisma, ${ }^{1}$ N. Boonsatit $\left(\mathbb{D},{ }^{2}\right.$ P. Hammachukiattikul $\left(\mathbb{D},{ }^{3}\right.$ \\ and G. Rajchakit $\mathbb{D}^{4}$ \\ ${ }^{1}$ Department of Mathematics, Sacred Heart College (Autonomous), Tirupattur, Vellore 635 601, Tamilnadu, India \\ ${ }^{2}$ Department of Mathematics, Faculty of Science and Technology, Rajamangala University of Technology Suvarnabhumi, \\ Nonthaburi 11000, Thailand \\ ${ }^{3}$ Department of Mathematics, Faculty of Science and Technology, Phuket Rajabhat University, Phuket 83000, Thailand \\ ${ }^{4}$ Department of Mathematics, Faculty of Science, Maejo University, Chiang Mai 50290, Thailand
}

Correspondence should be addressed to N. Boonsatit; nattakan.b@rmutsb.ac.th

Received 24 February 2021; Revised 19 March 2021; Accepted 5 April 2021; Published 30 April 2021

Academic Editor: Broumi Said

Copyright (C 2021 D. Ajay et al. This is an open access article distributed under the Creative Commons Attribution License, which permits unrestricted use, distribution, and reproduction in any medium, provided the original work is properly cited.

\begin{abstract}
Hypersoft set is a generalization of soft sets, which takes into account a multiargument function. The main objective of this work is to introduce fuzzy semiopen and closed hypersoft sets and study some of their characterizations and also to present neutrosophic semiopen and closed hypersoft sets, an extension of fuzzy hypersoft sets, along with few basic properties. We propose two algorithms based on neutrosophic hypersoft open sets and topology to obtain optimal decisions in MAGDM. The efficiency of the algorithms proposed is demonstrated by applying them to the current COVID-19 scenario.
\end{abstract}

\section{Introduction}

Fuzzy set theory [1] is an important tool for dealing with vagueness and incomplete data and is much more evolving and applied in different fields. Fuzzy set, which is an extension of general sets, has elements with membership function within the interval $[0,1]$. In view of other options of human thinking, fuzzy set along with some conditions is extended to the intuitionistic fuzzy set [2]. The intuitionistic fuzzy set assigns membership and nonmembership functions to each object which satisfies the constraint that the sum of both membership functions is between 0 and 1 .

Fuzziness was improved and extended from intuitionistic sets to neutrosophic sets. Smarandache [3] proposed neutrosophic sets, an essential mathematical tool which deals with incomplete, indeterminant, and inconsistent information. Neutrosophic set is characterized by the elements with truth, indeterminacy, and false membership functions which assume values within the range of 0 and 1. Wang et al. [4] proposed the concept of single-valued neutrosophic sets, a generalization of intuitionistic sets and a subclass of neutrosophic sets, which comprise elements with three membership functions which they belong to interval $[0,1]$. Under this neutrosophic environment, many researchers have worked on their extensions and developed many applications and results. A ranking approach based on the outranking relations of simplified neutrosophic numbers is developed in order to solve MCDM problems. Practical examples are provided to illustrate the proposed approach with a comparison analysis [5]. A comparison analysis is performed for this method with two examples [6], and the developed singlevalued neutrosophic TOPSIS extension is demonstrated on a numerical illustration of the evaluation and selection of e-commerce development strategies [7].

Molodtsov [8] introduced the idea of soft theory as a new approach to dealing with uncertainty, and now, there is a 
rapid growth of soft theory along with applications in various fields. Maji et al. [9] defined various basic concepts of soft theory, and the study of soft semirings by using the soft set theory has been initiated, and the notions of soft semirings, soft sub-semirings, soft ideals, idealistic soft semirings, and soft semiring homomorphisms with several related properties are investigated [10, 11]. Maji et al. [12] developed the fuzzy soft set theory, which is a combination of soft and fuzzy sets.

The idea of soft sets was generalized into hypersoft sets by Smarandache [13] by transforming the argument function $F$ into a multiargument function. He also introduced many results on hypersoft sets. Saqlain et al. [14] utilized this notion and proposed a generalized TOPSIS method for decision-making. Neutrosophic sets [15], from their very introduction, have seen many such extensions and have been very successful in applications. A new hybrid methodology for the selection of offshore wind power station location combining the Analytical Hierarchy Process and Preference Ranking Organization Method for Enrichment Evaluations methods in the neutrosophic environment has been proposed [16], a neutrosophic preference ranking organization method for enrichment evaluation technique for multicriteria decision-making problems to describe fuzzy information efficiently was proposed and applied to a real case study to select proper security service for FMEC in the presence of fuzzy information [17], and a model is proposed based on a plithogenic set and is applied to differentiate between COVID-19 and other four viral chest diseases under the uncertainty environment [18].

In 2019, Rana et al. [19] introduced the plithogenic fuzzy hypersoft set (PFHS) in the matrix form and defined some operations on the PFHS. Single- and multivalued neutrosophic hypersoft sets were proposed by Saqlain et al. [20], who also defined tangent similarity measure for singlevalued sets and an application of the same in a decisionmaking scenario. In another effort, Saqlain et al. [21] also introduced aggregation operators for neutrosophic hypersoft sets. A recent development in this area of research is the introduction of basic operations on hypersoft sets in which hypersoft points in different fuzzy environments are also introduced [22].

Fuzzy topology, a collection of fuzzy sets fulfilling the axioms, was defined by Chang [23]. A new definition of fuzzy space compactness and observed to have $\alpha$-compactness along with a Tychonoff theorem for an arbitrary product of $\alpha$-compact fuzzy spaces and a 1-point compactification [24], filters in the lattice $I^{X}$, where $I$ is the unit interval and $X$ an arbitrary set, have all been studied and using this study the convergence is defined in fuzzy topological space which leads to characterise fuzzy continuity and compactness [25]. Then, the basic concepts of intuitionistic fuzzy topological spaces were constructed, and the definitions of fuzzy continuity, fuzzy compactness, fuzzy connectedness, and fuzzy Hausdorff space and some characterizations concerning fuzzy compactness and fuzzy connectedness were defined [26]. Neutrosophic topological spaces were introduced by Salama and
Alblowi [27], and further concepts such as connectedness, semiclosed sets, and generalized closed sets [28] were developed.

The concept of fuzzy soft topology and some of its structural properties such as neighborhood of a fuzzy soft set, interior fuzzy soft set, fuzzy soft basis, and fuzzy soft subspace topology were studied [29]. The soft topological spaces, soft continuity of soft mappings, soft product topology, and soft compactness, as well as properties of soft projection mappings, have all been defined [30], and a relationship between a fuzzy soft set's closure and its fuzzy soft limit points has been constructed on fuzzy soft topological spaces [31]. Subspace, separation axioms, compactness, and connectedness on intuitionistic fuzzy soft topological spaces were defined along with some base theorems [32], some important properties of intuitionistic fuzzy soft topological spaces and intuitionistic fuzzy soft closure and interior of an intuitionistic fuzzy soft set were introduced, and an intuitionistic fuzzy soft continuous mapping with structural characteristics was studied [33]. A topology on a neutrosophic soft set was constructed, neutrosophic soft interior, neutrosophic soft closure, neutrosophic soft neighbourhood, and neutrosophic soft boundary were introduced, some of their basic properties were studied, and the concept of separation axioms on the neutrosophic soft topological space was introduced [34]. The concept of fuzzy hypersoft sets was applied to fuzzy topological spaces, and fuzzy hypersoft topological spaces were presented by Ajay and Charisma in [35]. In the same work, fuzzy hypersoft topology has been extended to intuitionistic and neutrosophic hypersoft topological spaces along with their properties. In this paper, we define the idea of semiopen sets in fuzzy hypersoft topological spaces with their characterization and extend to semiopen sets in intuitionistic and neutrosophic hypersoft topological spaces.

The paper is structured as follows: Section 2 recalls few basic terminologies and definitions of fuzzy hypersoft topological spaces. In Section 3, we define semiopen sets in fuzzy hypersoft topological spaces along with some of their properties. Sections 4 and 5 elaborate the logical extension of fuzzy hypersoft semiopen sets to intuitionistic and neutrosophic hypersoft semiopen sets. In Section 6 , we present an application of the neutrosophic hypersoft open set and topology in an MAGDM and conclude in Section 7.

\section{Preliminaries}

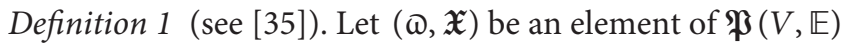
(where $\mathfrak{X}=X_{1} x X_{2} x X_{3} \ldots x X_{n}$ with each $X_{i}$ a subset of $\left.E_{i}(i=1,2, \ldots, n)\right)$, and let the set of all fuzzy hypersoft $(\mathrm{FH})$ subsets of $(\mathfrak{\Phi}, \mathfrak{X})$ be $P(\mathfrak{\omega}, \mathfrak{X})$ and $\tau$, a subcollection of

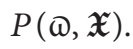

(i) $\varnothing_{\mathfrak{X}},(\emptyset, \mathfrak{X}) \in \tau$

(ii) $(\Theta, \mathfrak{J}),(\chi, \mathfrak{B}) \in \tau \Longrightarrow(\Theta, \mathfrak{J}) \cap(\chi, \mathfrak{B}) \in \tau$

(iii) $\left\{(\Theta, \mathfrak{\Im})_{l} \mid l \in L\right\} \in \tau \Longrightarrow \cup_{l \in L}(\Theta, \mathfrak{\Im})_{l} \in \tau$ 
If the above axioms are satisfied, then $\tau$ is a fuzzy hypersoft topology $(\mathrm{FHT})$ on $(\varpi, \mathfrak{X}) \cdot\left(\mathfrak{X}_{\oplus}, \tau\right)$ which is called a fuzzy hypersoft topological space (FHTS). Every member of $\tau$ is called an open fuzzy hypersoft set (OFHS). A fuzzy hypersoft set is said to be a closed fuzzy hypersoft set (CFHS) if its complement is OFHS.

Example 1. Let $V=\left\{x_{1}, x_{2}, x_{3}, x_{4}\right\}$ and the attributes be $E_{1}=\left\{a_{1}, a_{2}\right\}, E_{2}=\left\{a_{3}, a_{4}\right\}$, and $E_{3}=\left\{a_{5}, a_{6}\right\}$. Then, the fuzzy hypersoft set is

$$
\begin{aligned}
& \left(\left(a_{1}, a_{3}, a_{5}\right),\left\{\frac{x_{2}}{0.4}, \frac{x_{4}}{0.6}\right\}\right), \\
& \left(\left(a_{1}, a_{3}, a_{6}\right),\left\{\frac{x_{1}}{0.7}\right\}\right), \\
& \left(\left(a_{1}, a_{4}, a_{5}\right),\left\{\frac{x_{1}}{0.4}, \frac{x_{2}}{0.3}\right\}\right), \\
& \left(\left(a_{1}, a_{4}, a_{6}\right),\left\{\frac{x_{1}}{0.5}, \frac{x_{3}}{0.7}\right\}\right), \\
& \left(\left(a_{2}, a_{3}, a_{5}\right),\left\{\frac{x_{2}}{0.3}, \frac{x_{3}}{0.5}\right\}\right), \\
& \left(\left(a_{2}, a_{3}, a_{6}\right),\left\{\frac{x_{3}}{0.8}\right\}\right), \\
& \left(\left(a_{2}, a_{4}, a_{5}\right),\left\{\frac{x_{4}}{0.9}\right\}\right), \\
& \left(\left(a_{2}, a_{4}, a_{6}\right),\left\{\frac{x_{2}}{0.6}\right\}\right) .
\end{aligned}
$$

Let us consider this fuzzy hypersoft as $(\mathfrak{\emptyset}, \mathfrak{X})$. Then, the subfamily

$$
\begin{aligned}
& \tau=\varnothing_{\mathfrak{X}},(\bigoplus, \mathfrak{X}), \\
& \left\{\begin{array}{c}
\left(\left(a_{1}, a_{3}, a_{5}\right),\left\{\frac{x_{1}}{0.3}, \frac{x_{2}}{0.6}\right\}\right),\left(\left(a_{2}, a_{3}, a_{5}\right),\left\{\frac{x_{2}}{0.4}, \frac{x_{3}}{0.5}\right\}\right),\left(\left(a_{1}, a_{3}, a_{5}\right),\left\{\frac{x_{2}}{0.4}\right\}\right),\left(\left(a_{2}, a_{3}, a_{5}\right),\left\{\frac{x_{2}}{0.3}, \frac{x_{3}}{0.5}\right\}\right) \\
\left(\left(a_{1}, a_{3}, a_{5}\right),\left\{\frac{x_{1}}{0.3}, \frac{x_{2}}{0.6}, \frac{x_{3}}{0.6}\right\}\right),\left(\left(a_{1}, a_{4}, a_{6}\right),\left\{\frac{x_{1}}{0.5}, \frac{x_{3}}{0.7}\right\}\right),\left(\left(a_{1}, a_{3}, a_{6}\right),\left\{\frac{x_{1}}{0.7}\right\}\right),\left(\left(a_{1}, a_{4}, a_{5}\right),\left\{\frac{x_{1}}{0.4}, \frac{x_{2}}{0.3}\right\}\right), \\
\left(\left(a_{2}, a_{3}, a_{5}\right),\left\{\frac{x_{2}}{0.4}, \frac{x_{3}}{0.5}\right\}\right),\left(\left(a_{2}, a_{3}, a_{6}\right),\left\{\frac{x_{3}}{0.8}\right\}\right)\left(\left(a_{2}, a_{4}, a_{5}\right),\left\{\frac{x_{4}}{0.9}\right\}\right)\left(a_{2}, a_{4}, a_{6}\right),\left\{\frac{x_{2}}{0.6}\right\},
\end{array}\right\}
\end{aligned}
$$

of $P(\emptyset, \mathfrak{X})$ is a FHT on $(@, \mathfrak{X})$.

Definition 2 (see [35]). Let $\tau$ be a FHT on $(\emptyset, \mathfrak{X}) \in \mathfrak{P}(V, \mathbb{E})$ and $(\chi, \mathfrak{B})$ be a FH set in $P(\emptyset, \mathfrak{X})$. A FH set $(\Theta, \mathfrak{J})$ in $P(\emptyset, \mathfrak{X})$ is a neighbourhood of the FH set of $(\chi, \mathfrak{B})$ if and only if there exists an OFHS $(\xi, \mathfrak{C})$ such that $(\chi, \mathfrak{B}) \subset(\xi, \mathfrak{S}) \subset(\Theta, \mathfrak{I})$.

Definition 3 (see [35]). Let $\left(\mathfrak{X}_{\oplus}, \tau\right)$ be a FHTS and $(\Theta, \mathfrak{F}),(\chi, \mathfrak{B})$ be $\mathrm{FH}$ sets in $P(\mathfrak{\oplus}, \mathfrak{X})$ such that $(\chi, \mathfrak{B}) \subset(\Theta, \mathfrak{J})$. Then, $(\chi, \mathfrak{B})$ is said to be an interior fuzzy hypersoft set (IFHS) of $(\Theta, \mathfrak{J})$ if and only if $(\Theta, \mathfrak{J})$ is a neighbourhood of $(\chi, \mathfrak{B})$. The union of the whole IFHS of $(\Theta, \mathfrak{\Im})$ is named the interior of $(\Theta, \mathfrak{J})$ and denoted as $(\Theta, \mathfrak{J})^{\circ}$ or $\operatorname{FHint}(\Theta, \mathfrak{J})$.

Definition 4 (see [35]). Let $\left(\mathfrak{X}_{\infty}, \tau\right)$ be a FHTS and $(\Theta, \mathfrak{J}) \in \mathfrak{P}(V, \mathbb{E})$. The fuzzy hypersoft closure (FHC) of $(\Theta, \mathfrak{J})$ is the intersection of all CFH sets that contain $(\Theta, \mathfrak{J})$ which is denoted by $\overline{(\Theta, \mathfrak{J})}$ or $\operatorname{FHcl}(\Theta, \mathfrak{J})$.

Definition 5 (see [35]). Let $(₫, \mathfrak{X})$ be an element of $\mathfrak{P}(V, \mathbb{E})$ (where $\mathfrak{X}=X_{1} x X_{2} x X_{3} \ldots x X_{n}$ with each $X_{i}$ a subset of $E_{i}(i=1,2, \ldots, n)$. Let the set of all neutrosophic hypersoft 
(NH) subsets of $(\bowtie, \mathfrak{X})$ be $P(\emptyset, \mathfrak{X})$ and $\tau$, a subcollection of $P(\bowtie, \mathfrak{X})$.

$$
\begin{aligned}
& \text { (i) } \varnothing_{\mathfrak{X}},(@, \mathfrak{X}) \in \tau \\
& \text { (ii) }(\Theta, \mathfrak{J}),(\chi, \mathfrak{B}) \in \tau \Longrightarrow(\Theta, \mathfrak{J}) \cap(\chi, \mathfrak{B}) \in \tau \\
& \text { (iii) }\left\{(\Theta, \mathfrak{J})_{l} \mid l \in L\right\} \in \tau \Longrightarrow \cup_{l \in L}(\Theta, \mathfrak{J})_{l} \in \tau
\end{aligned}
$$

If the above axioms are satisfied, then $\tau$ is a neutrosophic hypersoft topology (NHT) on $(\bigoplus, \mathfrak{X}) \cdot\left(\mathfrak{X}_{\oplus}, \tau\right)$ which is called a neutrosophic hypersoft topological space (NHTS). Every member of $\tau$ is called an open neutrosophic hypersoft set (ONHS). A neutrosophic hypersoft set is called a closed neutrosophic hypersoft set (CNHS) if its complement is an ONHS.

For example, $\left\{\varnothing_{\mathfrak{X}},(\varpi, \mathfrak{X})\right\}$ and $P(\emptyset, \mathfrak{X})$ are neutrosophic hypersoft topologies on $(₫, \mathfrak{X})$ and are called indiscrete NHT and discrete NHT, respectively.

Definition 6 (see [35]). Let $\tau$ be a NHT on $(@, \mathfrak{X}) \in \mathfrak{P}(V, \mathbb{E})$ and $(\chi, \mathfrak{B})$ be a NH set in $P(\mathscr{\complement}, \mathfrak{X})$. A NH set $(\Theta, \mathfrak{J})$ in $P(\emptyset, \mathfrak{X})$ is a neighbourhood of the NH set of $(\chi, \mathfrak{B})$ iff there exists an ONHS $(\xi, \mathfrak{E})$ such that $(\chi, \mathfrak{B}) \subset(\xi, \mathfrak{C}) \subset(\Theta, \mathfrak{J})$.

Definition 7 (see [35]). Let $\left(\mathfrak{X}_{\oplus}, \tau\right)$ be a NHTS and $(\Theta, \mathfrak{I}),(\chi, \mathfrak{B})$ be $\mathrm{NH}$ sets in $\mathrm{P}(\mathfrak{\oplus}, \mathfrak{X})$ such that $(\chi, \mathfrak{B}) \subset(\Theta, \mathfrak{J})$. Then, $(\chi, \mathfrak{B})$ is said to be an interior neutrosophic hypersoft set (INHS) of $(\Theta, \mathfrak{J})$ if and only if
$(\Theta, \mathfrak{J})$ is a neighbourhood of $(\chi, \mathfrak{B})$. The union of the whole INHS of $(\Theta, \mathfrak{J})$ is named the interior of $(\Theta, \mathfrak{I})$ and denoted as $(\Theta, \mathfrak{\Im})^{\circ}$ or $\operatorname{FHint}(\Theta, \mathfrak{\Im})$.

Definition 8 (see [35]). Let $\left(\mathfrak{X}_{\omega}, \tau\right)$ be a NHTS and $(\Theta, \mathfrak{J}) \in \mathfrak{P}(V, \mathbb{E})$. The neutrosophic hypersoft closure (NHC) of $(\Theta, \mathfrak{\Im})$ is the intersection of all $\mathrm{CNH}$ sets that contain $(\Theta, \mathfrak{J})$ which is denoted by $\overline{(\Theta, \mathfrak{I})}$ or $\operatorname{FHcl}(\Theta, \mathfrak{J})$. Thus, $\overline{(\Theta, \mathfrak{I})}$ is the smallest CNHS which has $(\Theta, \mathfrak{\Im})$, and $\overline{(\Theta, \mathfrak{J})}$ is the CNHS.

\section{Fuzzy Semiopen and Closed Hypersoft Sets}

Definition 9. Let $\left(\mathfrak{X}_{\oplus}, \tau\right)$ be a FHTS and $(\Theta, \mathfrak{\Im}) \in P(\emptyset, \mathfrak{X})$. If $(\Theta, \mathfrak{J}) \subseteq \operatorname{FHcl}(\operatorname{FHint}(\Theta, \mathfrak{J}))$, then $(\Theta, \mathfrak{J})$ is called the fuzzy semiopen hypersoft set (FSOHS). We denote the set of all fuzzy semiopen hypersoft sets by FSOHS $(\mathfrak{X})$.

Definition 10. A fuzzy hypersoft set $(\Theta, \mathfrak{J})$ in the FHST space is a fuzzy semiclosed hypersoft set (FSCHS) if and only if its complement $(\Theta, \mathfrak{J})^{C}$ is FSOHS. The class of FSCHS is denoted by FSCHS $(\mathfrak{X})$.

Example 2. Let $X=\left\{y_{1}, y_{2}, y_{3}\right\}$ and the attributes be $E_{1}=\left\{a_{1}, a_{2}, a_{3}\right\}, E_{2}=\left\{b_{1}, b_{2}\right\}$, and $E_{3}=\left\{c_{1}, c_{2}\right\}$.

The fuzzy hypersoft topological space is $\tau$ :

$$
\begin{aligned}
\tau & =\left\{\left\{\left\langle\left(a_{1}, b_{1}, c_{2}\right),\left\{\frac{y_{1}}{0.9}, \frac{y_{2}}{0.2}, \frac{y_{3}}{0.2}\right\}\right\rangle,\left\langle\left(a_{1}, b_{2}, c_{2}\right)\left\{\frac{y_{1}}{0.8}, \frac{y_{2}}{0.7}, \frac{y_{3}}{0.4}\right\}\right\rangle\right\},\right. \\
& \left\{\left\langle\left(a_{1}, b_{1}, c_{1}\right),\left\{\frac{y_{1}}{0.8}, \frac{y_{2}}{0.6}, \frac{y_{3}}{0.3}\right\}\right\rangle\left\langle\left(a_{3}, b_{1}, c_{1}\right),\left\{\frac{y_{1}}{0.6}, \frac{y_{2}}{0.5}, \frac{y_{3}}{0.7}\right\}\right\rangle,\right. \\
& \left\{\left\langle\left(a_{1}, b_{1}, c_{2}\right)\left\{\frac{y_{1}}{0.9}, \frac{y_{2}}{0.2}, \frac{y_{3}}{0.2}\right\}\right\rangle,\left\langle\left(a_{1}, b_{2}, c_{2}\right),\left\{\frac{y_{1}}{0.8}, \frac{y_{2}}{0.7}, \frac{y_{3}}{0.4}\right\}\right\rangle\left\langle\left(a_{1}, b_{1}, c_{1}\right),\left\{\frac{y_{1}}{0.8}, \frac{y_{2}}{0.6}, \frac{y_{3}}{0.3}\right\}\right\rangle,\left\langle\left(a_{3}, b_{1}, c_{1}\right)\left\{\frac{y_{1}}{0.6}, \frac{y_{2}}{0.5}, \frac{y_{3}}{0.7}\right\}\right\rangle\right\} .
\end{aligned}
$$

The fuzzy hypersoft set

$$
\left\{\begin{array}{c}
\left\langle\left(a_{1}, b_{1}, c_{2}\right),\left\{\frac{y_{1}}{0.9}, \frac{y_{2}}{0.3}, \frac{y_{3}}{0.5}\right\}\right\rangle,\left\langle\left(a_{1}, b_{2}, c_{2}\right),\left\{\frac{y_{1}}{0.9}, \frac{y_{2}}{0.8}, \frac{y_{3}}{0.5}\right\}\right\rangle, \\
\left\langle\left(a_{1}, b_{1}, c_{1}\right),\left\{\frac{y_{1}}{0.9} \frac{y_{2}}{0.7}, \frac{y_{3}}{0.5}\right\}\right\rangle,\left\langle\left(a_{3}, b_{1}, c_{1}\right),\left\{\frac{y_{1}}{0.7}, \frac{y_{2}}{0.6}, \frac{y_{3}}{0.8}\right\}\right\rangle
\end{array}\right\}
$$

is a FSOHS.

Theorem 1. Let $\left(\mathfrak{X}_{\oplus}, \tau\right)$ be a FHTS and $(\Theta, \mathfrak{J}) \in \operatorname{FSOHS}(\mathfrak{X})$; then,

(i) Arbitrary fuzzy hypersoft union of FSOHS is FSOHS

(ii) Arbitrary fuzzy hypersoft intersection of FSCHS is FSCHS

Proof

(i) Let $\left\{(\Theta, \mathfrak{J})_{j}: j \in J\right\} \subseteq \mathrm{FSOHS}(\mathfrak{X})$.
Then, $\forall j \in J,(\Theta, \mathfrak{J})_{j} \subseteq \operatorname{FHcl}(\Theta, \mathfrak{J})_{j}$.

Hence, $\quad \cup_{j}(\Theta, \mathfrak{\Im})_{j} \subseteq \bigcup\{$ FHclFHint $\}(\Theta, \mathfrak{J})_{j} \subseteq$ FHcl $\left(\right.$ FHint $\left.\left(\cup_{j}(\Theta, \mathfrak{\Im})_{j}\right)\right)$.

Therefore, $\cup_{j}(\Theta, \mathfrak{J})_{j} \in \operatorname{FSOHS}(\mathfrak{X})$.

Similarly, (ii) is proved.

Theorem 2. Let $\left(\mathfrak{X}_{\oplus}, \tau\right)$ be a FHTS and $(\Theta, \mathfrak{\Im}) \in P(\emptyset, \mathfrak{X})$. Then,

(i) $(\Theta, \mathfrak{J}) \in F S O H S(\mathfrak{X})$ if and only if there exists $(\chi, \mathfrak{B}) \in \tau$ such that $(\chi, \mathfrak{B}) \subseteq(\Theta, \mathfrak{\Im}) \subseteq F H c l(\chi, \mathfrak{B})$

(ii) If $(\Theta, \mathfrak{J}) \in \operatorname{FSOHS}(\mathfrak{X})$ and $(\Theta, \mathfrak{J}) \subseteq(\xi, \mathfrak{E}) \subseteq$ $\operatorname{FHcl}(\Theta, \mathfrak{I})$, then $(\xi, \mathfrak{E}) \in \operatorname{FSOHS}(\mathfrak{X})$

Proof

(i) Let $(\Theta, \mathfrak{J}) \in \operatorname{FSOHS}(\mathfrak{X})$.

Then, $(\Theta, \mathfrak{J}) \subseteq \operatorname{FHcl}(\operatorname{FHint}(\Theta, \mathfrak{J}))$.

We know that $\operatorname{FHint}(\Theta, \mathfrak{I}) \subseteq(\Theta, \mathfrak{I})$; thus, FHint $(\Theta, \mathfrak{J}) \subseteq(\Theta, \mathfrak{J}) \subseteq \operatorname{FHcl}(\operatorname{FHint}(\Theta, \mathfrak{J}))$. 
Let $(\chi, \mathfrak{B})=$ FHint $(\Theta, \mathfrak{I})$; thus, we get $(\chi, \mathfrak{B}) \subseteq$ $(\Theta, \mathfrak{J}) \subseteq \operatorname{FHcl}(\chi, \mathfrak{B})$.

Conversely, let $(\chi, \mathfrak{B}) \subseteq(\Theta, \mathfrak{J}) \subseteq \operatorname{FHcl}(\chi, \mathfrak{B})$ for some $(\chi, \mathfrak{B}) \in \tau$. Then, $(\chi, \mathfrak{B}) \subseteq$ FHint $(\Theta, \mathfrak{J}) \subseteq(\Theta, \mathfrak{J})$.

$\Longrightarrow \operatorname{FHcl}(\chi, \mathfrak{B}) \subseteq \operatorname{FHcl}(\operatorname{FHint}(\Theta, \mathfrak{\Im}))$.

Thus, $(\Theta, \mathfrak{J}) \subseteq \operatorname{FHcl}(\operatorname{FHint}(\Theta, \mathfrak{J}))$.

Therefore, $(\Theta, \mathfrak{J}) \in \operatorname{FSOHS}(\mathfrak{X})$.

(ii) Let $(\Theta, \mathfrak{J}) \in \operatorname{FSOHS}(\mathfrak{X})$. Then, for some $(\chi, \mathfrak{B}) \in$ $\tau,(\chi, \mathfrak{B}) \subseteq(\Theta, \mathfrak{J}) \subseteq \operatorname{FHcl}(\chi, \mathfrak{B})$. If $\quad(\chi, \mathfrak{B}) \subseteq(\Theta, \mathfrak{J}) \subseteq$ $(\xi, \mathfrak{S})$, then $(\chi, \mathfrak{B}) \subseteq(\xi, \mathfrak{S}) \subseteq \operatorname{FHcl}(\Theta, \mathfrak{\Im}) \subseteq \operatorname{FHcl}(\chi, \mathfrak{B})$.

Hence, $\quad(\chi, \mathfrak{B}) \subseteq(\xi, \mathfrak{E}) \subseteq \operatorname{FHcl}(\chi, \mathfrak{B})$. Thus, by (i), $(\xi, \mathfrak{C}) \in \operatorname{FSOHS}(\mathfrak{X})$.

Definition 11. Let $\left(\mathfrak{X}_{\oplus}, \tau\right)$ be a FHTS and $(\Theta, \mathfrak{J}) \in P(\emptyset, \mathfrak{X})$.

Then, the largest fuzzy semiopen hypersoft set contained in $(\Theta, \mathfrak{J})$ is called the fuzzy semi-hypersoft interior of $(\Theta, \mathfrak{J})$ and denoted by $\operatorname{FSHSint}(\Theta, \mathfrak{J})$, i.e., FSHSint $(\Theta$,

$\mathfrak{J})=\cup\{(\chi, \mathfrak{B}):(\chi, \mathfrak{B}) \subseteq(\Theta, \mathfrak{S})(\chi, \mathfrak{B}) \in \operatorname{FSOHS}(\mathfrak{X})\}$.

And the smallest fuzzy semiclosed hypersoft set containing $(\Theta, \mathfrak{J})$ is called the fuzzy semi-hypersoft closure of $(\Theta, \mathfrak{J})$ and denoted by FSHScl $(\Theta, \mathfrak{J})$.

$\operatorname{FSHScl}(\Theta, \mathfrak{J})=\cap\{(\xi, \mathfrak{C}):(\xi, \mathfrak{C}) \supseteq(\Theta, \mathfrak{J})$, and $(\xi, \mathfrak{S}) \in$ $\operatorname{FSCHS}(\mathfrak{X})$.

Theorem 3. Let $\left(\mathfrak{X}_{\oplus}, \tau\right)$ be a FHTS and $(\Theta, \mathfrak{I}),(\chi$, $\mathfrak{B}) \in P(\mathfrak{⿴ 囗 \mathfrak { X } )})$. Then, the following properties hold:

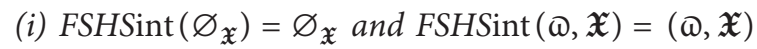

(ii) $\operatorname{FSHSint}(\Theta, \mathfrak{\Im}) \subseteq(\Theta, \mathfrak{J})$

(iii) FSHS int $(\Theta, \mathfrak{I})$ is the largest fuzzy semiopen hypersoft set contained in $(\Theta, \mathfrak{J})$

(iv) If $(\Theta, \mathfrak{J}) \subseteq(\chi, \mathfrak{B})$, then $F \operatorname{FHSint}(\Theta, \mathfrak{J}) \subseteq$ FSHSint $(\chi, \mathfrak{B})$

(v) $F H S H \operatorname{int}(F H S H \operatorname{int}(\Theta, \mathfrak{J}))=F H S H \operatorname{Hint}(\Theta, \mathfrak{J})$

(vi) FHSHint $(\Theta, \mathfrak{\Im}) \cup F H S H i n t(\chi, \quad \mathfrak{B}) \subseteq F H S H i n t$ $[(\Theta, \mathfrak{\Im}) \cup(\chi, \mathfrak{B})]$

(vii) $\operatorname{FHSHint}[(\Theta, \mathfrak{I}) \cap(\chi, \quad \mathfrak{B})] \subseteq F H S H \operatorname{Hint}(\Theta, \mathfrak{J}) \cap$ FHSHint $(\chi, \mathfrak{B})$

Theorem 4. Let $\left(\mathfrak{X}_{\oplus}, \tau\right)$ be a FHTS and $(\Theta, \mathfrak{J}),(\chi, \mathfrak{B}) \in P(\emptyset, \mathfrak{X})$. Then, the following properties hold:

(i) $\operatorname{FSHScl}\left(\varnothing_{\mathfrak{X}}\right)=\varnothing_{\mathfrak{X}}$, and $\operatorname{FSHScl}(@, \mathfrak{X})=(@, \mathfrak{X})$

(ii) $\operatorname{FSHScl}(\Theta, \mathfrak{J}) \subseteq(\Theta, \mathfrak{J})$

(iii) $\operatorname{FSHScl}(\Theta, \mathfrak{J})$ is the smallest fuzzy semiclosed hypersoft set that contains $(\Theta, \mathfrak{J})$

(iv) If $(\Theta, \mathfrak{\Im}) \subseteq(g, B)$, then $F S H S c l(\Theta, \mathfrak{J}) \subseteq F S H S c l(\chi, \mathfrak{B})$

(v) $\operatorname{FHSHcl}(\operatorname{FHSHcl}(\Theta, \mathfrak{J}))=\operatorname{FHSHcl}(\Theta, \mathfrak{J})$

(vi) $\operatorname{FHSHcl}(\Theta, \mathfrak{\Im}) \cup \mathrm{FHSHcl}(\chi, \mathfrak{B}) \subseteq F H S H c l(\Theta, \mathfrak{\Im}) \cup$ $[(\chi, \mathfrak{B})]$

(vii) $\operatorname{FHSHcl}[(\Theta, \mathfrak{I}) \cap(\chi, \mathfrak{B})] \subseteq F H S H c l(\Theta, \quad \mathfrak{J}) \cap$ $\operatorname{FHSHcl}(\chi, \mathfrak{B})$
Theorem 5. Every fuzzy open (closed) hypersoft set in a FHTS $\left(\mathfrak{X}_{\oplus}, \tau\right)$ is a fuzzy semiopen (closed) hypersoft set.

Proof. Let $(\Theta, \mathfrak{\Im})$ be a fuzzy open hypersoft set. Then, $\operatorname{FHint}(\Theta, \mathfrak{J})=(\Theta, \mathfrak{J}) . \quad$ Since $\quad(\Theta, \mathfrak{J}) \subseteq \operatorname{FHcl}(\Theta, \mathfrak{J})$, $(\Theta, \mathfrak{J}) \subseteq \operatorname{FHcl}(\operatorname{FHint}(\Theta, \mathfrak{J}))$. Thus, $(\Theta, \mathfrak{J}) \in \operatorname{FSOHS}(\mathfrak{X})$.

Theorem 6. Let $\left(\mathfrak{X}_{\oplus}, \tau\right)$ be a FHTS and $(\Theta, \mathfrak{J}),(\chi$, $\mathfrak{B}) \in P(\emptyset, \mathfrak{X})$. If either $(\Theta, \mathfrak{J}) \in F \operatorname{FOHS}(X)$ or $(\chi, \mathfrak{B}) \in F S O H S(\mathfrak{X})$, then $F H c l(F H i n t(\Theta, \mathfrak{J}) \cap(\chi, \mathfrak{B}))=$ $\operatorname{FHcl}(F H i n t(\Theta, \mathfrak{J}) \cap F H c l F H i n t(\chi, \mathfrak{B}))$.

Proof. Let $(\Theta, \mathfrak{J}),(\chi, \mathfrak{B}) \in P(\emptyset, \mathfrak{X})$.

Then, we have

$$
\begin{aligned}
& \operatorname{FHcl}(\operatorname{FHint}(\Theta, \mathfrak{J}) \cap(\chi, \mathfrak{B})) \subseteq \\
& \operatorname{FHcl}(\operatorname{FHint}(\Theta, \mathfrak{J}) \cap \operatorname{FHclFHint}(\chi, \mathfrak{B})) \\
& \operatorname{FHcl}(\operatorname{FHint}(\Theta, \mathfrak{J}) \cap \operatorname{FHclFHint}(\chi, \mathfrak{B})) \subseteq \\
& \operatorname{FHcl}[\operatorname{FHcl}(\operatorname{FHint}(\Theta, \mathfrak{J})) \cap \operatorname{FHcl}(\operatorname{FHint}(\chi, \mathfrak{B}))] \\
& =\operatorname{FHcl}[\operatorname{FHcl}[\operatorname{FHint}(\Theta, \mathfrak{J}) \cap \operatorname{FHint}(\chi, \mathfrak{B})]] \\
& =\operatorname{FHcl}[\operatorname{FHcl}[\operatorname{FHint}[(\Theta, \mathfrak{\Im}) \cap(\chi, \mathfrak{B})]]] \\
& \subseteq \operatorname{FHcl}[\operatorname{FHint}(\Theta, \mathfrak{J}) \cap(\chi, \mathfrak{B})] \\
& \Longrightarrow \operatorname{FHcl}(\operatorname{FHint}(\Theta, \mathfrak{J})) \cap \operatorname{FHcl}(\operatorname{FHint}(\chi, \mathfrak{B})) \\
& \subseteq \operatorname{FHcl}(\operatorname{FHint}(\Theta, \mathfrak{J}) \cap(\chi, \mathfrak{B})) .
\end{aligned}
$$

Thus, $\quad \operatorname{FHcl}(\operatorname{FHint}(\Theta, \mathfrak{\Im}) \cap(\chi, \mathfrak{B}))=$ FHclFHint $(\Theta, \mathfrak{J}) \cap \operatorname{FHcl}(\operatorname{FHint}(\chi, \mathfrak{B}))$.

Theorem 7. Let $\left(\mathfrak{X}_{\oplus}, \tau\right)$ be a FHTS, $(\Theta, \mathfrak{I})$ be a fuzzy hypersoft open set, and $(\chi, \mathfrak{B}) \in \operatorname{FSOHS}(\mathfrak{X})$. Then, $(\Theta, \mathfrak{J}) \cap(\chi, \mathfrak{B}) \in \operatorname{FSOHS}(\mathfrak{X})$.

Proof. Let $(\Theta, \mathfrak{I})$ be a FOHS and $(\chi, \mathfrak{B})$ be a FSOHS. Then, $\quad(\Theta, \mathfrak{J}) \cap(\chi, \mathfrak{B}) \supseteq$ FHint $((\Theta, \mathfrak{J}) \cap(\chi, \mathfrak{B})) \Longrightarrow$ FHint $((\Theta, \mathfrak{J}) \cap(\chi, \mathfrak{B})) \subseteq(\Theta, \mathfrak{J}) \cap(\chi, \mathfrak{B})$.

Then, $\quad$ FHint $((\Theta, \mathfrak{J}) \cap(\chi, \mathfrak{B})) \subseteq(\Theta, \mathfrak{J}) \cap(\chi, \mathfrak{B}) \backslash \subseteq$ FHclFHint $((\Theta, \mathfrak{\Im}) \cap(\chi, \mathfrak{B})) \quad$ FHint $((\Theta, \mathfrak{\Im}) \cap(\chi, \mathfrak{B})) \subseteq$ $(\Theta, \mathfrak{J}) \cap(\chi, \mathfrak{B}) \backslash \subseteq$ FHclFHint $((\Theta, \mathfrak{J}) \cap(\chi, \mathfrak{B}))(\Theta, \mathfrak{J}) \cap(\chi$, $\mathfrak{B}) \subseteq$ FHclFHint $((\Theta, \mathfrak{\Im}) \cap(\chi, \mathfrak{B}))$.

Therefore, $(\Theta, \mathfrak{J}) \cap(\chi, \mathfrak{B})$ is a FSOHS.

Proposition 1. Let $(\Theta, \mathfrak{J})$ be a fuzzy hypersoft set in the FHTS $\left(\mathfrak{X}_{\oplus}, \tau\right)$. Then, $(\Theta, \mathfrak{J})$ is the FSCHS if and only if there exists an FCHS set $(\xi, \mathfrak{J})$ such that $\operatorname{FHint}(\xi, \mathfrak{\Im}) \subseteq(\Theta, \mathfrak{\Im}) \subseteq(\xi, \mathfrak{\Im})$.

Proposition 2. Every fuzzy hypersoft closed set is a FSCHS in a FHTS $\left(\mathfrak{X}_{\infty}, \tau\right)$, but the converse need not be true.

Theorem 8. Let $(\Theta, \mathfrak{\Im})$ be a FHS in a FHTS $\left(\mathfrak{X}_{\oplus}, \tau\right)$. Then, $(\Theta, \mathfrak{J})$ is a FSCHS if and only if FHint $(F H c l(\Theta, \mathfrak{J})) \subseteq(\Theta, \mathfrak{J})$.

Proof. Suppose $(\Theta, \mathfrak{J})$ is a FSCHS. Then, there exists a $\operatorname{FHCS}(\Theta, \mathfrak{J})$ such that $\operatorname{FHint}(\xi, \mathfrak{J}) \subseteq(\Theta, \mathfrak{J}) \subseteq(\xi, \mathfrak{J})$. $\operatorname{FHcl}(\Theta, \mathfrak{J}) \subseteq \operatorname{FHcl}(\xi, \mathfrak{J})=(\xi, \mathfrak{J})$. 
Thus, $\quad \operatorname{FHint}(\operatorname{FHcl}(\Theta, \mathfrak{J})) \subseteq \operatorname{FHint}(\xi, \mathfrak{J}) \subseteq(\Theta, \mathfrak{J}) \Longrightarrow$ $\operatorname{FHint}(\operatorname{FHcl}(\xi, \mathfrak{J})) \subseteq(\Theta, \mathfrak{\Im})$.

Conversely, let $(\Theta, \mathfrak{I})$ be a fuzzy hypersoft set in $\left(\mathfrak{X}_{\mathfrak{\Phi}}, \tau\right)$ such that FHint $(\operatorname{FHcl}(\Theta, \mathfrak{\Im})) \subseteq(\Theta, \mathfrak{\Im})$. Let $\operatorname{FHcl}(\Theta, \mathfrak{J})=(\xi, \mathfrak{J})$. Then, $\operatorname{FHint}(\Theta, \mathfrak{J}) \subseteq(\Theta, \mathfrak{J}) \subseteq(\xi, \mathfrak{J})$. Thus, $(\Theta, \mathfrak{J})$ is a FSCHS.

Theorem 9. Let $\left\{(\Theta, \mathfrak{\Im})_{\beta}: \beta \in I\right\}$ be a family of FSCHS in a FHTS $\left(\mathfrak{X}_{\omega}, \tau\right)$. Then, the intersection $\cap_{\beta \in I}(\Theta, \mathfrak{J})_{\beta}$ is a FSCHS in $\left(\mathfrak{X}_{\mathscr{Q}}, \tau\right)$.

Proof. Since each $\beta \in \mathrm{I},(\Theta, \mathfrak{\Im})_{\beta}$ is a FSCHS. Then, there exists a $\operatorname{FCHS}(\xi, \mathfrak{J})_{\beta}$ such that $\operatorname{FHint}\left((h, A)_{\beta}\right) \subseteq$ $(\Theta, \mathfrak{J})_{\beta} \subseteq(\xi, \mathfrak{J})_{\beta}$. Thus, $\quad \cap_{\beta \in I}\left(\operatorname{FHint}\left((\xi, \mathfrak{J})_{\beta}\right)\right) \subseteq \cap_{\beta \in I}$ $(\Theta, \mathfrak{J})_{\beta} \subseteq \cap_{\beta \in I}(\xi, \mathfrak{J})_{\beta}$. Consider $\cap_{\beta \in I}(\xi, \mathfrak{J})_{\beta}=(\xi, \mathfrak{J})$. Then, $(\xi, \mathfrak{J})$ is a FCHS, and hence, $\cap_{\beta \in I}(\Theta, \mathfrak{J})_{\beta}$ is a FSCHS.

Theorem 10. Let $(\Theta, \mathfrak{\Im})$ be a FSCHS and $(\vartheta, \mathfrak{\Im})$ be a FCHS in $\left(\mathfrak{X}_{\oplus}, \tau\right)$. If FHint $(\Theta, \mathfrak{I}) \subseteq(\vartheta, \mathfrak{J}) \subseteq(\Theta, \mathfrak{J})$, then $(\Theta, \mathfrak{J})$ is a FSCHS.

Proof. Since $(\Theta, \mathfrak{J})$ is a FSCHS, there exists a FCHS $(\xi, \mathfrak{J})$ such that $\operatorname{FHint}(\xi, \mathfrak{J}) \subseteq(\Theta, \mathfrak{J}) \subseteq(\xi, \mathfrak{J})$. Then, $(\vartheta, \mathfrak{J}) \subseteq(\xi, \mathfrak{J})$. Also, $\quad \operatorname{FHintFHint}(\xi, \mathfrak{J}) \subseteq$ FHint $(\xi, \mathfrak{J}) \subseteq$ FHint $(\Theta, \mathfrak{J}) \Longrightarrow$ $\operatorname{FHint}(\xi, \mathfrak{J}) \subseteq(\vartheta, \mathfrak{J})$. Therefore, $\operatorname{FHint}(\xi, \mathfrak{I}) \subseteq(\vartheta, \mathfrak{J}) \subseteq(\xi, \mathfrak{J})$. Hence, $(\Theta, \mathfrak{J})$ is a FSCHS.

Remark 1. For any FCHS $(\Theta, \mathfrak{J}), \operatorname{FSHScl}(\Theta, \mathfrak{J})=(\Theta, \mathfrak{J})$. And for any $\operatorname{FOHS}(\zeta, \mathfrak{J}), \operatorname{FSHSint}(\zeta, \mathfrak{J})=(\zeta, \mathfrak{J})$.

Remark 2. If $(\Theta, \mathfrak{I})$ is a fuzzy hypersoft set in $\left(\mathfrak{X}_{\mathfrak{⿴}}, \tau\right)$, then FHint $(\Theta, \mathfrak{J}) \subseteq$ FSHSint $\quad(\Theta, \mathfrak{J}) \subseteq(\Theta, \mathfrak{J}) \subseteq \operatorname{FSHScl}(\Theta, \mathfrak{J}) \subseteq$ $\operatorname{FHcl}(\Theta, \mathfrak{\Im})$.

Theorem 11. Let $(\Theta, \mathfrak{J})$ be a FHS in $\left(\mathfrak{X}_{\oplus}, \tau\right)$. Then,

(i) $(\operatorname{FSHSint}(\Theta, \mathfrak{\Im}))^{C}=\operatorname{FSHScl}\left((\Theta, \mathfrak{\Im})^{C}\right)$

(ii) $\operatorname{FSHScl}(\Theta, \mathfrak{\Im}))^{C}=\operatorname{FSHSint}\left((\Theta, \mathfrak{\Im})^{C}\right)$

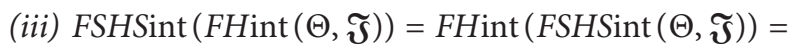
$\operatorname{FHint}(\Theta, \mathfrak{\Im})$

(iv) $\operatorname{FSHScl}(\operatorname{FHcl}(\Theta, \mathfrak{\Im}))=\operatorname{FHcl}(\operatorname{FSHScl}(\Theta, \mathfrak{\Im})) \backslash \backslash=$ $\operatorname{FHcl}(\Theta, \mathfrak{\Im})$ (i) $\operatorname{FSHSint}(\Theta, \mathfrak{J}) \subseteq(\Theta, \mathfrak{J}) \Longrightarrow(\Theta, \mathfrak{J})^{C} \subseteq(\operatorname{FSHSint}(\Theta$, $\mathfrak{J}))^{C}$.

Since $(\operatorname{FSHSint}(\Theta, \mathfrak{\Im}))^{C}$ is a $\operatorname{FSCHS}, F S H S c l(\Theta$, $\mathfrak{\Im})^{C} \subseteq \operatorname{FSHScl}\left((\mathrm{FSHSint}(\Theta, \mathfrak{\Im}))^{C}\right)=(\operatorname{FSHSint}(\Theta$, J) $)^{C}$.

Conversely, $\quad(\Theta, \mathfrak{\Im})^{C} \subseteq$ FSHScl $\left((\Theta, \mathfrak{\Im})^{C}\right) \Longrightarrow$ $\operatorname{FSHScl}\left((\Theta, \mathfrak{J})^{C}\right)^{C} \subseteq\left((\Theta, \mathfrak{J})^{C}\right)^{C}=(\Theta, \mathfrak{J})$.

$\operatorname{FSHScl}\left((\Theta, \mathfrak{J})^{\mathrm{C}}\right)$ being FSCHS implies that $\operatorname{FSHScl}\left((\Theta, \mathfrak{J})^{C}\right)^{C}$ is a FSOHS set.

Thus, FSHScl $\left((\Theta, \mathfrak{\Im})^{C}\right)^{C} \subseteq$ FSHSint $(\Theta, \mathfrak{\Im})$.

And hence, $\quad(\operatorname{FSHSint}(\Theta, \mathfrak{\Im}))^{C} \subseteq$ $\left(\operatorname{FSHScl}\left((\Theta, \mathfrak{\Im})^{C}\right)^{C}\right)^{C}=\left(\operatorname{FSHScl}\left((\Theta, \mathfrak{J})^{C}\right)\right)$.

(ii) The proof is the same as that of (i).

(iii) FHint $(\Theta, \mathfrak{\Im})$ is FOHS implying that it is FSOHS. Therefore, FSHSint $(\operatorname{FHint}(\Theta, \mathfrak{J}))=\operatorname{FHint}(\Theta, \mathfrak{J})$. $\operatorname{Now}, \operatorname{FHint}(\Theta, \mathfrak{J}) \subseteq$ FSHSint $(\Theta, \mathfrak{I})=(\Theta, \mathfrak{J})$. Thus, FSHSint $(\operatorname{FHint}(\Theta, \mathfrak{J}))=\operatorname{FHint}(\Theta, \mathfrak{J})$.

(iv) $\mathrm{FHcl}(\Theta, \mathfrak{J})$ is fuzzy closed hypersoft implying that is FSCHS. Therefore, $\operatorname{FSHScl}(\operatorname{FHcl}(\Theta, \mathfrak{\Im}))=$ $\operatorname{FHcl}(\Theta, \mathfrak{\Im}) . \quad$ Now, $\quad(\Theta, \mathfrak{\Im}) \subseteq \operatorname{FSHScl}(\Theta, \mathfrak{\Im}) \subseteq$ $\operatorname{FHcl}(\Theta, \mathfrak{\Im})$.

Hence,

$\operatorname{FSHScl}(\Theta, \mathfrak{\Im}) \subseteq \operatorname{FHclFSHScl}((\Theta, \mathfrak{\Im})) \subseteq$ $\operatorname{FSHScl}(\Theta, \mathfrak{J})$.

This implies $\operatorname{IHcl}(\operatorname{ISHScl}(\Theta, \mathfrak{\Im})) \subseteq \operatorname{IHcl}(\Theta, \mathfrak{\Im})$.

\section{Intuitionistic Semiopen and Closed Hypersoft Sets}

Definition 12. Let $\left(\mathfrak{X}_{\oplus}, \tau\right)$ be an IHTS and $(\Theta, \mathfrak{I}) \in P(\Theta, \mathfrak{X})$. If $(\Theta, \mathfrak{J}) \subseteq \operatorname{IHcl}(\operatorname{IHint}(\Theta, \mathfrak{I}))$, then $(\Theta, \mathfrak{J})$ is called an intuitionistic semiopen hypersoft set (ISOHS). We denote the set of all intuitionistic semiopen hypersoft sets by ISOHS $(\mathfrak{X})$.

Definition. 13An intuitionistic hypersoft set $(\Theta, \mathfrak{J})$ in the IHST space is an intuitionistic semiclosed hypersoft set (ISCHS) if and only if its complement $(\Theta, \mathfrak{I})^{C}$ is ISOHS. The class of ISCHS is denoted by ISCHS $(\mathfrak{X})$.

Example 3. Let $X=\left\{y_{1}, y_{2}, y_{3}\right\}$ and the attributes be $E_{1}=\left\{a_{1}, a_{2}, a_{3}\right\}, E_{2}=\left\{b_{1}, b_{2}\right\}$, and $E_{3}=\left\{c_{1}, c_{2}\right\}$.

The intuitionistic hypersoft topological space is $\tau$ :

$$
\begin{aligned}
\tau= & \left\{\left\langle\left(a_{1}, b_{1}, c_{2}\right),\left\{\frac{y_{1}}{0.9,0.1}, \frac{y_{2}}{0.2,0.4}, \frac{y_{3}}{0.2,0.7}\right\}\right\rangle,\left\langle\left(a_{1}, b_{2}, c_{2}\right),\left\{\frac{y_{1}}{0.8,0.3}, \frac{y_{2}}{0.7,0.2}, \frac{y_{3}}{0.4,0.8}\right\}\right\rangle,\right. \\
& \cdot\left\{\left\langle\left(a_{1}, b_{1}, c_{1}\right)\left\{\frac{y_{1}}{0.8,0.2}, \frac{y_{2}}{0.6,0.4}, \frac{y_{3}}{0.3,0.2}\right\}\right\rangle,\left\langle\left(a_{3}, b_{1}, c_{1}\right),\left\{\frac{y_{1}}{0.6,0.5}, \frac{y_{2}}{0.5,0.4} \frac{y_{3}}{0.7,0.2}\right\}\right\rangle\right\},
\end{aligned}
$$




$$
\left\{\begin{array}{c}
\left\langle\left(a_{1}, b_{1}, c_{2}\right),\left\{\frac{y_{1}}{0.9,0.1}, \frac{y_{2}}{0.2,0.4}, \frac{y_{3}}{0.2,0.7}\right\}\right\rangle, \\
\left\langle\left(a_{1}, b_{2}, c_{2}\right),\left\{\frac{y_{1}}{0.8,0.3}, \frac{y_{2}}{0.7,0.2}, \frac{y_{3}}{0.4,0.8}\right\}\right\rangle,\left\langle\left(a_{1}, b_{1}, c_{1}\right)\left\{\frac{y_{1}}{0.8,0.2}, \frac{y_{2}}{0.6,0.4}, \frac{y_{3}}{0.3,0.2}\right\}\right\rangle, \\
\left\langle\left(a_{3}, b_{1}, c_{1}\right),\left\{\frac{y_{1}}{0.6,0.5} \frac{y_{2}}{0.5,0.4}, \frac{y_{3}}{0.7,0.2}\right\}\right\rangle
\end{array}\right\} .
$$

The intuitionistic hypersoft set

$$
\left\{\begin{array}{c}
\left\langle\left(a_{1}, b_{1}, c_{2}\right)\left\{\frac{y_{1}}{0.9,0.1}, \frac{y_{2}}{0.3,0.3}, \frac{y_{3}}{0.5,0.5}\right\}\right\rangle,\left\langle\left(a_{1}, b_{2}, c_{2}\right)\left\{\frac{y_{1}}{0.9,0.2}, \frac{y_{2}}{0.8,0.1}, \frac{y_{3}}{0.5,0.7,0.5}\right\}\right\rangle, \\
\left\langle\left(a_{1}, b_{1}, c_{1}\right),\left\{\frac{y_{1}}{0.9,0.1}, \frac{y_{2}}{0.7,0.3}, \frac{y_{3}}{0.5,0.1}\right\}\right\rangle,\left\langle\left(a_{3}, b_{1}, c_{1}\right),\left\{\frac{y_{1}}{0.7,0.3}, \frac{y_{2}}{0.6,0.3}, \frac{y_{3}}{0.8,0.1}\right\}\right\rangle
\end{array}\right\}
$$

is ISOHS.

Theorem 12. Let $\left(\mathfrak{X}_{\oplus}, \tau\right)$ be an IHTS and $(\Theta, \mathfrak{J}) \in \operatorname{ISOHS}(\mathfrak{X})$; then,

(i) Arbitrary intuitionistic hypersoft union of ISOHS is an ISOHS

(ii) Arbitrary intuitionistic hypersoft intersection of ISCHS is an ISCHS

Proof

(i) Let $\left\{(\Theta, \mathfrak{\Im})_{j}: j \in J\right\} \subseteq \operatorname{ISOHS}(\mathfrak{X})$.

Then, $\forall j \in \mathrm{J},(\Theta, \mathfrak{J})_{j} \subseteq \operatorname{FHcl}(\Theta, \mathfrak{J})_{j}$.

Hence, $\quad \cup_{j}(\Theta, \mathfrak{\Im})_{j} \subseteq \cup \operatorname{IHc}\left(\operatorname{IHint}(\Theta, \mathfrak{\Im})_{j}\right) \subseteq \operatorname{IHcl}$

$\left(\operatorname{IHint}\left(\cup_{j}(\Theta, \mathfrak{\Im})_{j}\right)\right)$.

Therefore, $\cup_{j}(\Theta, \mathfrak{J})_{j} \in \operatorname{ISOHS}(\mathfrak{X})$.

Similarly, (ii) is proved.

Theorem 13. Let $\left(\mathfrak{X}_{\oplus}, \tau\right)$ be an IHTS and $(\Theta, \mathfrak{\Im}) \in P(\emptyset, \mathfrak{X})$. Then,

(i) $(\Theta, \mathfrak{F}) \in \operatorname{ISOHS}(\mathfrak{X})$ if and only if there exists $(\chi, \mathfrak{B}) \in \tau$ such that $(\chi, \mathfrak{B}) \subseteq(\Theta, \mathfrak{\Im}) \subseteq \operatorname{IHcl}(\chi, \mathfrak{B})$

(ii) If $(\Theta, \mathfrak{J}) \in \operatorname{ISOHS}(\mathfrak{X}) \quad$ and $\quad(\Theta, \mathfrak{J}) \subseteq(\xi$, $\mathfrak{(}) \subseteq \operatorname{Icl}(\Theta, \mathfrak{J})$, then $(\xi, \mathfrak{E}) \in \operatorname{ISOHS}(\mathfrak{X})$

Proof

(i) Let $(\Theta, \mathfrak{J}) \in \operatorname{ISOHS}(\mathfrak{X})$. Then, $\quad(\Theta, \mathfrak{J}) \subseteq$ $\operatorname{IHcl}(\operatorname{IHint}(\Theta, \mathfrak{\Im}))$. We know that $\operatorname{IHint}(\Theta, \mathfrak{J}) \subseteq(\Theta, \mathfrak{\Im})$; $\quad$ thus, $\operatorname{IHint}(\Theta, \mathfrak{J}) \subseteq(\Theta, \mathfrak{J}) \subseteq \operatorname{IHcl}(\operatorname{IHint}(\Theta, \mathfrak{J}))$. Let $(\chi, \mathfrak{B})=\operatorname{IHint}(\Theta, \mathfrak{J})$; thus, we get $(\chi, \mathfrak{B}) \subseteq$ $(\Theta, \mathfrak{J}) \subseteq \operatorname{IHcl}(\chi, \mathfrak{B})$.
Conversely, let $(\chi, \mathfrak{B}) \subseteq(\Theta, \mathfrak{\Im}) \subseteq \operatorname{IHcl}(\chi, \mathfrak{B})$ for some $(\chi, \mathfrak{B}) \in \tau . \quad$ Then, $\quad(\chi, \mathfrak{B}) \subseteq \operatorname{IHint}(\Theta, \mathfrak{\Im}) \subseteq(\Theta, \mathfrak{F})$. $\Longrightarrow \operatorname{IHcl}(\chi, \mathfrak{B}) \subseteq \operatorname{IHcl}(\operatorname{IHint}(\Theta, \mathfrak{\Im}))$.

Thus, $(\Theta, \mathfrak{J}) \subseteq \operatorname{IHcl}(\operatorname{IHint}(\Theta, \mathfrak{J}))$.

Therefore, $(\Theta, \mathfrak{I}) \in \operatorname{ISOHS}(\mathfrak{X})$.

(ii) Let $(\Theta, \mathfrak{J}) \in \operatorname{ISOHS}(\mathfrak{X})$. Then, for some $(\chi, \mathfrak{B}) \in$ $\tau,(\chi, \mathfrak{B}) \subseteq(\Theta, \mathfrak{\Im}) \subseteq \operatorname{IHcl}(\chi, \mathfrak{B})$. If $\quad(\chi, \mathfrak{B}) \subseteq(\Theta, \mathfrak{J}) \subseteq$ $(\xi, \mathfrak{S})$, then $(\chi, \mathfrak{B}) \subseteq(\xi, \mathfrak{C}) \subseteq \operatorname{IHcl}(\Theta, \mathfrak{J}) \subseteq \operatorname{IHcl}(\chi, \mathfrak{B})$. Hence, $\quad(\chi, \mathfrak{B}) \subseteq(\xi, \mathfrak{C}) \subseteq \operatorname{IHcl}(\chi, \mathfrak{B})$. Thus, by (i), $(\xi, \mathfrak{S}) \in \operatorname{ISOHS}(\mathfrak{X})$.

Definition 14. Let $\left(\mathfrak{X}_{\oplus}, \tau\right)$ be an IHTS and $(\Theta, \mathfrak{\Im}) \in$ $P(@, \mathfrak{X})$.

Then, the largest intuitionistic semiopen hypersoft set contained in $(\Theta, \mathfrak{J})$ is called the intuitionistic semihypersoft interior of $(\Theta, \mathfrak{J})$ and denoted by $\operatorname{ISHSint}(\Theta, \mathfrak{\Im})$, i.e., $\quad \operatorname{ISHSint}(\Theta, \mathfrak{I})=\cup\{(\chi, \mathfrak{B}):(\chi, \mathfrak{B}) \subseteq(\Theta, \mathfrak{J}),(\chi, \mathfrak{B}) \in$ $\operatorname{ISOHS}(\mathfrak{X})\}$.

And the smallest intuitionistic semiclosed hypersoft set containing $(\Theta, \mathfrak{J})$ is called the intuitionistic semi-hypersoft closure of $(\Theta, \mathfrak{J})$ and denoted by $\operatorname{ISHScl}(\Theta, \mathfrak{J})$. $\operatorname{ISHScl}(\Theta, \mathfrak{I})=\cap\{(\xi, \mathfrak{E}):(\xi, \mathfrak{C}) \supseteq(\Theta, \mathfrak{J}) \quad$ and $(\xi, \mathfrak{E}) \in \operatorname{ISCHS}(\mathfrak{X})$.

Theorem 14. Let $\left(\mathfrak{X}_{\oplus}, \tau\right)$ be an IHTS and $(\Theta, \mathfrak{J}),(\chi, \mathfrak{B}) \in P(\mathfrak{\Phi}, \mathfrak{X})$. Then, the following properties hold:

(i) $\operatorname{ISHSint}\left(\varnothing_{\mathfrak{X}}\right)=\varnothing_{\mathfrak{X}}$ and $\operatorname{ISHSint}(\emptyset, \mathfrak{X})=(@, \mathfrak{X})$

(ii) $\operatorname{ISHSint}(\Theta, \mathfrak{J}) \subseteq(\Theta, \mathfrak{J})$

(iii) ISHSint $(\Theta, \mathfrak{\Im})$ is the largest intuitionistic semiopen hypersoft set contained in $(\Theta, \mathfrak{J})$

(iv) If $(\Theta, \mathfrak{I}) \subseteq(\chi, \mathfrak{B}), \quad$ then $\operatorname{ISHSint}(\Theta$, $\mathfrak{J}) \subseteq I S H \operatorname{Sint}(\chi, \mathfrak{B})$

(v) $\operatorname{IHSHint}(\operatorname{IHSHint}(\Theta, \mathfrak{J}))=\operatorname{IHSHint}(\Theta, \mathfrak{J})$ 
(vi) $\operatorname{IHSHint}(\Theta, \mathfrak{\Im}) \cup I H S H i n t(\chi, \mathfrak{B}) \subseteq I H S H i n t$ $[(\Theta, \mathfrak{J}) \cup(\chi, \mathfrak{B})]$

(vii) $\operatorname{IHSHint}[(\Theta, \mathfrak{\Im}) \cap(\chi, \mathfrak{B})] \subseteq \operatorname{IHSHint}(\Theta, \mathfrak{J}) \cap$ IHSHint $(\chi, \mathfrak{B})$

Theorem 15. Let $\left(\mathfrak{X}_{\oplus}, \tau\right)$ be an IHTS and $(\Theta, \mathfrak{J}),(\chi, \mathfrak{B}) \in P(\Theta, \mathfrak{X})$. Then, the following properties hold:

(i) $\operatorname{ISHScl}\left(\varnothing_{\mathfrak{X}}\right)=\varnothing_{\mathfrak{X}}$ and $\operatorname{ISHScl}(\bowtie, \mathfrak{X})=(\bowtie, \mathfrak{X})$

(ii) $\operatorname{ISHScl}(\Theta, \mathfrak{J}) \subseteq(\Theta, \mathfrak{\Im})$

(iii) $\operatorname{ISHScl}(\Theta, \mathfrak{\Im})$ is the smallest intuitionistic semiclosed hypersoft set that contains $(\Theta, \mathfrak{\Im})$

(iv) If $(\Theta, \mathfrak{\Im}) \subseteq(g, B)$, then $\operatorname{ISHScl}(\Theta, \mathfrak{J}) \subseteq \operatorname{ISHScl}(\chi, \mathfrak{B})$

(v) $\operatorname{IHSHcl}(\operatorname{IHSHcl}(\Theta, \mathfrak{\Im}))=\operatorname{IHSHcl}(\Theta, \mathfrak{\Im})$

(vi) $\operatorname{IHSHcl}(\Theta, \mathfrak{\Im}) \cup \operatorname{IHSHcl}(\chi, \mathfrak{B}) \subseteq \operatorname{IHSHcl}[(\Theta, \mathfrak{\Im}) \cup($ $\chi, \mathfrak{B})]$

(vii) $\operatorname{IHSHcl}[(\Theta, \mathfrak{\Im}) \cap(\chi, \mathfrak{B})] \subseteq \operatorname{IHSHcl}(\Theta, \mathfrak{\Im}) \cap I H S H c l$ $(\chi, \mathfrak{B})$

Theorem 16. Every intuitionistic open (closed) hypersoft set in an IHTS $\left(\mathfrak{X}_{\mathfrak{\omega}}, \tau\right)$ is an intuitionistic semiopen (closed) hypersoft set.

Proof. Let $(\Theta, \mathfrak{\Im})$ be an intuitionistic open hypersoft set. Then, $\operatorname{IHint}(\Theta, \mathfrak{J})=(\Theta, \mathfrak{J})$. Since $(\Theta, \mathfrak{I}) \subseteq \operatorname{IHcl}(\Theta, \mathfrak{J})$, $(\Theta, \mathfrak{J}) \subseteq \operatorname{IHcl}(\operatorname{IHint}(\Theta, J))$. Thus, $(\Theta, \mathfrak{J}) \in \operatorname{ISOHS}(\mathfrak{X})$.

Theorem 17. Let $\left(\mathfrak{X}_{\oplus}, \tau\right)$ be an IHTS and $(\Theta, \mathfrak{J}),(\chi, \mathfrak{B}) \in P(\Phi, \mathfrak{X})$. If either $(\Theta, \mathfrak{J}) \in \operatorname{ISOHS}(X)$ or $(\chi, \mathfrak{B}) \in \operatorname{ISOHS}(\mathfrak{X}), \quad$ then $\operatorname{IHcl}(\operatorname{IHint}(\Theta, \mathfrak{J}) \cap(\chi, \mathfrak{B}))=$ $\operatorname{IHcl}(\operatorname{IHint}(\Theta, \mathfrak{\Im}) \cap \mathrm{FHclIHint}(\chi, \mathfrak{B}))$.

Proof. Let then, we have

$$
\begin{aligned}
& \operatorname{IHcl}(\operatorname{IHint}(\Theta, \mathfrak{J}) \cap(\chi, \mathfrak{B})) \subseteq \\
& \operatorname{IHcl}(\operatorname{IHint}(\Theta, \mathfrak{J}) \cap \operatorname{IclIHint}(\chi, \mathfrak{B})) \\
& \operatorname{FHcl}(\operatorname{IHint}(\Theta, \mathfrak{\Im}) \cap \operatorname{IHclIHint}(\chi, \mathfrak{B})) \subseteq \\
& \operatorname{IHcl}[\operatorname{IHcl}(\operatorname{IHint}(\Theta, \mathfrak{\Im})) \cap \operatorname{IHcl}(\operatorname{IHint}(\chi, \mathfrak{B}))] \\
& =\operatorname{IHcl}[\operatorname{IHcl}[\operatorname{IHint}(\Theta, \mathfrak{J}) \cap \operatorname{IHint}(\chi, \mathfrak{B})]] \\
& =\operatorname{IHcl}[\operatorname{IHcl}[\operatorname{IHint}[(\Theta, \mathfrak{\Im}) \cap(\chi, \mathfrak{B})]]] \\
& \subseteq \operatorname{IHcl}[\operatorname{IHint}(\Theta, \mathfrak{\Im}) \cap(\chi, \mathfrak{B})] \\
& \Longrightarrow \operatorname{IHcl}(\operatorname{IHint}(\Theta, \mathfrak{\Im})) \cap \operatorname{IHcl}(\operatorname{IHint}(\chi, \mathfrak{B})) \\
& \subseteq \operatorname{IHcl}(\operatorname{IHint}(\Theta, \mathfrak{\Im}) \cap(\chi, \mathfrak{B})) \text {. } \\
& \cdot(\Theta, \mathfrak{J}),(\chi, \mathfrak{B}) \in P(\bowtie, \mathfrak{X})
\end{aligned}
$$

Thus, $\operatorname{IHcl}(\operatorname{IHint}(\Theta, \mathfrak{\Im}) \cap(\chi, \mathfrak{B}))=\operatorname{IHclIHint}(\Theta, \mathfrak{I}) \cap$ $\operatorname{IHcl}(\operatorname{IHint}(\chi, \mathfrak{B}))$.

Theorem 18. Let $\left(\mathfrak{X}_{\mathscr{\omega}}, \tau\right)$ be an IHTS, $(\Theta, \mathfrak{J})$ be an intuitionistic hypersoft open set, and $(\chi, \mathfrak{B}) \in \operatorname{ISOHS}(\mathfrak{X})$. Then, $(\Theta, \mathfrak{I}) \cap(\chi, \mathfrak{B}) \in \operatorname{ISOHS}(\mathfrak{X})$.

Proof. Let $(\Theta, \mathfrak{\Im})$ be an IOHS and $(\chi, \mathfrak{B})$ be an ISOHS.
Then, $(\Theta, \mathfrak{\Im}) \cap(\chi, \mathfrak{B}) \supseteq \operatorname{IHint}((\Theta, \mathfrak{\Im}) \cap(?, \mathfrak{B})) \Longrightarrow$ IHint $((\Theta, \mathfrak{J}) \cap(\chi, \mathfrak{B})) \subseteq(\Theta, \mathfrak{I}) \cap(\chi, \mathfrak{B})$.

Then, $\quad \operatorname{IHint}((\Theta, \mathfrak{J}) \cap(\chi, \mathfrak{B})) \subseteq(\Theta, \mathfrak{I}) \cap(\chi, \mathfrak{B}) \subseteq$ IHclIHint $((\Theta, \mathfrak{J}) \cap(\chi, \mathfrak{B})) . \Longrightarrow(\Theta, \mathfrak{J}) \cap(\chi, \mathfrak{B}) \subseteq$ IHclIHint $((\Theta, \mathfrak{J}) \cap(\chi, \mathfrak{B}))$.

Therefore, $(\Theta, \mathfrak{J}) \cap(\chi, \mathfrak{B})$ is an ISOHS.

Proposition 3. Let $(\Theta, \mathfrak{\Im})$ be an intuitionistic hypersoft set in the IHTS $\left(\mathfrak{X}_{\mathscr{Q}}, \tau\right)$. Then, $(\Theta, \mathfrak{\Im})$ is ISCHS if and only if there exists an ICHS set $(\xi, \mathfrak{\Im})$ such that $\operatorname{IHint}(\xi, \mathfrak{\Im}) \subseteq(\Theta, \mathfrak{\Im}) \subseteq(\xi, \mathfrak{\Im})$

Proposition 4. Every intuitionistic hypersoft closed set is an ISCHS in an IHTS $\left(\mathfrak{X}_{\oplus}, \tau\right)$, but the converse need not be true.

Theorem 19. Let $(\Theta, \mathfrak{\Im})$ be an IHS in an IHTS $\left(\mathfrak{X}_{\varpi}, \tau\right)$. Then, $(\Theta, \mathfrak{\Im})$ is an ISCHS if and only if $\operatorname{IHint}(\operatorname{IHcl}(\Theta, \mathfrak{J})) \subseteq(\Theta, \mathfrak{J})$.

Proof. Suppose $(\Theta, \mathfrak{J})$ is an ISCHS; then, there exists an $\operatorname{IHCS}(\Theta, \mathfrak{J})$ such that $\operatorname{IHint}(\xi, \mathfrak{J}) \subseteq(\Theta, \mathfrak{J}) \subseteq(\xi, \mathfrak{J})$. $\operatorname{IHcl}(\Theta, \mathfrak{J}) \subseteq \operatorname{IHcl}(\xi, \mathfrak{\Im})=(\xi, \mathfrak{J})$.

Thus, $\quad \operatorname{IHint}(\operatorname{IHcl}(\Theta, \mathfrak{I})) \subseteq \operatorname{IHint}(\xi, \mathfrak{J}) \subseteq$ $(\Theta, \mathfrak{\Im}) \Longrightarrow \operatorname{IHint}(\operatorname{IHcl}(\xi, \mathfrak{J})) \subseteq(\Theta, \mathfrak{J})$.

Conversely, let $(\Theta, \mathfrak{J})$ be an intuitionistic hypersoft set in $\left(\mathfrak{X}_{?}, \tau\right)$ such that $\operatorname{IHint}(\operatorname{IHcl}(\Theta, \mathfrak{\Im})) \subseteq(\Theta, \mathfrak{\Im})$. Let $\operatorname{IHcl}(\Theta, \mathfrak{J})=(\xi, \mathfrak{J})$. Then, $\operatorname{IHint}(\Theta, \mathfrak{J}) \subseteq(\Theta, \mathfrak{J}) \subseteq(\xi, \mathfrak{J})$. Thus, $(\Theta, \mathfrak{J})$ is an ISCHS.

Theorem 20. Let $\left\{(\Theta, \mathfrak{\Im})_{\beta}: \beta \in I\right\}$ be a family of ISCHSs in an IHTS $\left(\mathfrak{X}_{\mathscr{\omega}}, \tau\right)$. Then, the intersection $\cup_{\beta \in I}(\Theta, \mathfrak{J})_{\beta}$ is an ISCHS in $\left(\mathfrak{X}_{\mathfrak{\omega}}, \tau\right)$.

Proof. Since each $\beta \in I,(\Theta, \mathfrak{\Im})_{\beta}$ is an ISCHS. Then, there exists an ICHS $(\xi, \mathfrak{J})_{\beta}$ such that $\operatorname{IHint}((h$, $\left.A)_{\beta}\right) \subseteq(\Theta, \mathfrak{J})_{\beta} \subseteq(\xi, \mathfrak{J})_{\beta}$.

Thus, $\cap_{\beta \in I}\left(\operatorname{IHint}\left((\xi, \mathfrak{J})_{\beta}\right)\right) \subseteq \cap_{\beta \in I}(\Theta, \mathfrak{J})_{\beta} \subseteq \cap_{\beta \in I}(\xi, \mathfrak{J})_{\beta}$. Consider $\cap_{\beta \in I}(\xi, \mathfrak{J})_{\beta}=(\xi, \mathfrak{J})$. Then, $(\xi, \mathfrak{J})$ is an ICHS, and hence, $\cap_{\beta \in I}(\Theta, \mathfrak{J})_{\beta}$ is an ISCHS.

Theorem 21. Let $(\Theta, \mathfrak{\Im})$ be an ISCHS and $(\vartheta, \mathfrak{\Im})$ be an ICHS in $\left(\mathfrak{X}_{\varrho}, \tau\right)$. If IHint $(\Theta, \mathfrak{J}) \subseteq(\vartheta, \mathfrak{J}) \subseteq(\Theta, \mathfrak{J})$, then $(\Theta, \mathfrak{J})$ is an ISCHS.

Proof. Since $(\Theta, \mathfrak{J})$ is an ISCHS, there exists an ICHS $(\xi, \mathfrak{J})$ such that $\operatorname{IHint}(\xi, \mathfrak{J}) \subseteq(\Theta, \mathfrak{J}) \subseteq(\xi, \mathfrak{J})$. Then, $(\vartheta, \mathfrak{J}) \subseteq(\xi, \mathfrak{J})$. Also, IHintIHint $(\xi, \mathfrak{J}) \subseteq \operatorname{IHint}(\xi, \mathfrak{J}) \subseteq \operatorname{IHint}(\Theta, \mathfrak{J}) \Longrightarrow$ IHint $(\xi, \mathfrak{J}) \subseteq(\vartheta, \mathfrak{J})$. Therefore, $\quad \operatorname{IHint}(\xi, \mathfrak{I}) \subseteq(\vartheta, \mathfrak{J}) \subseteq(\xi, \mathfrak{J})$. Hence, $(\Theta, \mathfrak{J})$ is an ISCHS.

Remark 3. For any ICHS $(\Theta, \mathfrak{J})$, $\operatorname{ISHScl}(\Theta, \mathfrak{J})=(\Theta, \mathfrak{J})$. And for any $\operatorname{IOHS}(\zeta, \mathfrak{J})$, ISHSint $(\zeta, \mathfrak{J})=(\zeta, \mathfrak{J})$.

Remark 4. If $(\Theta, \mathfrak{J})$ is an intuitionistic hypersoft set in $\left(\mathfrak{X}_{\oplus}, \tau\right)$, then $\operatorname{IHint}(\Theta, \mathfrak{J}) \subseteq \operatorname{ISHSint}(\Theta, \mathfrak{J}) \subseteq$ $(\Theta, \mathfrak{J}) \subseteq \operatorname{ISHScl}(\Theta, \mathfrak{I}) \subseteq \operatorname{IHcl}(\Theta, \mathfrak{J})$

Theorem 22. Let $(\Theta, \mathfrak{J})$ be an IHS in $\left(\mathfrak{X}_{\oplus}, \tau\right)$. Then, 
(i) $\operatorname{ISHSint}(\Theta, \mathfrak{\Im}))^{C}=\operatorname{ISHScl}\left((\Theta, \mathfrak{\Im})^{C}\right)$

(ii) $\operatorname{ISHScl}(\Theta, \mathfrak{J}))^{C}=\operatorname{ISHSint}\left((\Theta, \mathfrak{J})^{C}\right)$

(iii) $\operatorname{ISHSint}(\operatorname{IHint}(\Theta, \mathfrak{J}))=$

$I H \operatorname{Hint}(\operatorname{ISHSint}(\Theta, \mathfrak{\Im})) \backslash \backslash=I H \operatorname{Hint}(\Theta, \mathfrak{\Im})$

(iv) $\operatorname{ISHScl}(\operatorname{IHcl}(\Theta, \mathfrak{J}))=\operatorname{IHcl}(\operatorname{ISHScl}(\Theta, \mathfrak{\Im})) \backslash \backslash=$

$\operatorname{IHcl}(\Theta, \mathfrak{J})$

Proof

(i) $\operatorname{ISHSint}(\Theta, \mathfrak{J}) \subseteq(\Theta, \mathfrak{J}) \Longrightarrow(\Theta, \mathfrak{J})^{C} \subseteq(\operatorname{ISHSint}(\Theta$,

J) $)^{C}$.

Since $\operatorname{ISHSint}(\Theta, \mathfrak{\Im}))^{C}$ is an ISCHS, ISHScl $(\Theta$, $\mathfrak{\Im})^{C} \subseteq \operatorname{ISHScl}\left((\operatorname{FSHSint}(\Theta, \mathfrak{\Im}))^{C}\right)=(\operatorname{ISHSint}(\Theta$, $\mathfrak{J}))^{C}$.

Conversely, $\quad(\Theta, \mathfrak{I})^{C} \subseteq \operatorname{ISHScl}\left((\Theta, \mathfrak{I})^{C}\right) \Longrightarrow$ $\left(\operatorname{ISHScl}(\Theta, \mathfrak{J})^{C}\right)^{C} \subseteq\left((\Theta, \mathfrak{J})^{C}\right)^{C}=(\Theta, \mathfrak{J})$.

$\operatorname{ISHScl}\left((\Theta, \mathfrak{J})^{C}\right)$ being ISCHS implies that $\operatorname{ISHScl}\left((\Theta, \widetilde{J})^{C}\right)^{C}$ is an ISOHS set. Thus, $\operatorname{ISHScl}\left((\Theta, \mathfrak{J})^{C}\right)^{C} \subseteq \operatorname{ISHSint}(\Theta, \mathfrak{J})$. And hence, $(\operatorname{ISHSint}(\Theta, \mathfrak{\Im}))^{C} \subseteq(\operatorname{ISHScl}((\Theta$,

$\left.\left.(\mathfrak{J})^{C}\right)^{C}\right)^{C}=\left(\operatorname{ISHScl}\left((\Theta, \mathfrak{J})^{C}\right)\right)$.

(ii) The proof is the same as that of (i).

(iii) $\operatorname{IHint}(\Theta, \mathfrak{J})$ is IOHS which implies that it is ISOHS.

Therefore, $\operatorname{ISHSint}(\operatorname{IHint}(\Theta, \mathfrak{\Im}))=\operatorname{IHint}(\Theta, \mathfrak{J})$.

$\operatorname{Now}, \operatorname{IHint}(\Theta, \mathfrak{\Im}) \subseteq \operatorname{ISHSint}(\Theta, \mathfrak{J})=(\Theta, \mathfrak{J})$.
Thus, ISHSint $(\operatorname{IHint}(\Theta, \mathfrak{\Im}))=\operatorname{IHint}(\Theta, \mathfrak{\Im})$.

(iv) $\operatorname{IHcl}(\Theta, \mathfrak{J})$ is an intuitionistic closed hypersoft set, and this implies that it is an ISCHS. Therefore, $\operatorname{ISHScl}(\operatorname{IHcl}(\Theta, \mathfrak{\Im}))=\operatorname{IHcl}(\Theta, \mathfrak{J})$. $(\Theta, \mathfrak{J}) \subseteq \operatorname{ISHScl}(\Theta, \mathfrak{\Im}) \subseteq \operatorname{IHcl}(\Theta, \mathfrak{J})$.

Hence, $\quad \operatorname{ISHScl}(\Theta, \mathfrak{J}) \subseteq \operatorname{IHclISHScl}((\Theta, \mathfrak{J})) \subseteq$ ISHScl $(\Theta, \mathfrak{J})$

This implies $\operatorname{IHcl}(\operatorname{ISHScl}(\Theta, \mathfrak{\Im})) \subseteq \operatorname{IHcl}(\Theta, \mathfrak{\Im})$.

\section{Neutrosophic Semiopen and Closed Hypersoft Sets}

Definition 15. Let $\left(\mathfrak{X}_{\oplus}, \tau\right)$ be a NHTS and $(\Theta, \mathfrak{\Im}) \in P(\emptyset, \mathfrak{X})$. If $(\Theta, \mathfrak{J}) \subseteq \operatorname{NHcl}(\operatorname{NHint}(\Theta, \mathfrak{J}))$, then $(\Theta, \mathfrak{J})$ is called a neutrosophic semiopen hypersoft set (NSOHS). We represent the collection of all neutrosophic semiopen hypersoft sets by $\operatorname{NSOHS}(\mathfrak{X})$.

Definition 16. A neutrosophic hypersoft set $(\Theta, \mathfrak{J})$ in the NHST space is a neutrosophic semiclosed hypersoft set (NFSCHS) iff its complement $(\Theta, \mathfrak{J})^{\mathrm{C}}$ is NSOHS. The class of NSCHS is denoted by $\operatorname{NSCHS}(\mathfrak{X})$.

Example 4. Let $X=\left\{y_{1}, y_{2}, y_{3}\right\}$ and the attributes be $E_{1}=\left\{a_{1}, a_{2}, a_{3}\right\}, E_{2}=\left\{b_{1}, b_{2}\right\}$, and $E_{3}=\left\{c_{1}, c_{2}\right\}$.

The neutrosophic hypersoft topological space is

$$
\begin{aligned}
\tau=\{ & \left.\left\{\left\langle\left(a_{1}, b_{1}, c_{2}\right),\left\{\frac{y_{1}}{0.9,0.2,0.1}, \frac{y_{2}}{0.2,0.2,0.4}, \frac{y_{3}}{0.2,0.1,0.7}\right\}\right\rangle,\left\langle\left(a_{1}, b_{2}, c_{2}\right),\left\{\frac{y_{1}}{0.8,0.4,0.3}, \frac{y_{2}}{0.7,0.4,0.2}, \frac{y_{3}}{0.4,0.6,0.8}\right\}\right\rangle\right\}\right\} \\
& \cdot\left\{\left\langle\left(a_{1}, b_{1}, c_{1}\right),\left\{\frac{y_{1}}{0.8,0.5,0.2}, \frac{y_{2}}{0.6,0.5,0.4}, \frac{y_{3}}{0.3,0.6,0.2}\right\}\right\rangle,\left\langle\left(a_{3}, b_{1}, c_{1}\right),\left\{\frac{y_{1}}{0.6,0.4,0.5}, \frac{y_{2}}{0.5,0.7,0.4}, \frac{y_{3}}{0.7,0.3,0.2}\right\}\right\rangle\right\} \\
& \cdot\left\{\begin{array}{l}
\left\langle\left(a_{1}, b_{1}, c_{2}\right),\left\{\frac{y_{1}}{0.9,0.2,0.1}, \frac{y_{2}}{0.2,0.2,0.4}, \frac{y_{3}}{0.2,0.1,0.7}\right\}\right\rangle,\left\langle\left(a_{1}, b_{2}, c_{2}\right),\left\{\frac{y_{1}}{0.8,0.4,0.3}, \frac{y_{2}}{0.7,0.4,0.2}, \frac{y_{3}}{0.4,0.6,0.8}\right\}\right\rangle, \\
\left.\left\langle\left(a_{1}, b_{1}, c_{1}\right),\left\{\frac{y_{1}}{0.8,0.5,0.2}, \frac{y_{2}}{0.6,0.5,0.4}, \frac{y_{3}}{0.3,0.6,0.2}\right\}\right\rangle,\left\langle\left(a_{3}, b_{1}, c_{1}\right),\left\{\frac{y_{1}}{0.6,0.4,0.5}, \frac{y_{2}}{0.5,0.7,0.4}, \frac{y_{3}}{0.7,0.3,0.2}\right\}\right\rangle\right\} .
\end{array}\right.
\end{aligned}
$$

The neutrosophic hypersoft set

$$
\left\{\begin{array}{l}
\left\langle\left(a_{1}, b_{1}, c_{2}\right)\left\{\frac{y_{1}}{0.9,0.3,0.1}, \frac{y_{2}}{0.3,0.4,0.3}, \frac{y_{3}}{0.5,0.2,0.5}\right\}\right\rangle,\left\langle\left(a_{1}, b_{2}, c_{2}\right)\left\{\frac{y_{1}}{0.9,0.5,0.2}, \frac{y_{2}}{0.8,0.5,0.1}, \frac{y_{3}}{0.5,0.7,0.5}\right\}\right\rangle, \\
\left\langle\left(a_{1}, b_{1}, c_{1}\right)\left\{\frac{y_{1}}{0.9,0.6,0.1}, \frac{y_{2}}{0.7,0.7,0.3}, \frac{y_{3}}{0.5,0.7,0.1}\right\}\right\rangle,\left\langle\left(a_{3}, b_{1}, c_{1}\right)\left\{\frac{y_{1}}{0.7,0.5,0.3}, \frac{y_{2}}{0.6,0.8,0.3}, \frac{y_{3}}{0.8,0.4,0.1}\right\}\right\rangle
\end{array}\right\}
$$


is NSOHS.

Theorem 23. Let $\left(\mathfrak{X}_{\oplus}, \tau\right)$ be a NHTS and $(\Theta, \mathfrak{J}) \in \operatorname{NSOHS}(\mathfrak{X})$; then,

(i) Arbitrary neutrosophic hypersoft union of NSOHS is a NSOHS

(ii) Arbitrary neutrosophic hypersoft intersection of NSCHS is a NFSCHS

Proof

(i) Let $\left\{(\Theta, \mathfrak{J})_{j}: j \in J\right\} \subseteq \operatorname{NSOHS}(\mathfrak{X})$.

Then, $\forall j \in J,(\Theta, \mathfrak{J})_{j} \subseteq \operatorname{NHcl}(\Theta, \mathfrak{J})_{j}$.

Hence, $\quad \cup_{j}(\Theta, \mathfrak{J})_{j} \subseteq \cup \operatorname{NHcl}\left(\operatorname{NHint}(\Theta, \mathfrak{J})_{j}\right) \subseteq \backslash \backslash \mathrm{NHcl}$

$\left(\operatorname{NHint}\left(\cup_{j}(\Theta, \mathfrak{\Im})_{j}\right)\right)$.

Therefore, $\cup_{j}(\Theta, \mathfrak{F})_{j} \in \operatorname{NSOHS}(\mathfrak{X})$.

Similarly, (ii) is proved.

Theorem 24. Let $\left(\mathfrak{X}_{\oplus}, \tau\right)$ be a NHTS and $(\Theta, \mathfrak{J}) \in P(\emptyset, \mathfrak{X})$. Then,

(i) $(\Theta, \mathfrak{I}) \in \operatorname{NSOHS}(\mathfrak{X})$ if and only if there exists $(\chi, \mathfrak{B}) \in \tau$ such that $(\chi, \mathfrak{B}) \subseteq(\Theta, \mathfrak{J}) \subseteq N H c l(\chi, \mathfrak{B})$

(ii) If $(\Theta, \mathfrak{J}) \in \operatorname{NSOHS}(\mathfrak{X})$ and $(\Theta, \mathfrak{I}) \subseteq(\xi, \mathfrak{C}) \subseteq$ $\operatorname{Ncl}(\Theta, \mathfrak{J})$, then $(\xi, \mathfrak{S}) \in \operatorname{NSOHS}(\mathfrak{X})$

Proof

(i) Let $\quad(\Theta, \mathfrak{J}) \in \operatorname{NSOHS}(\mathfrak{X}) . \quad$ Then, $\quad(\Theta, \mathfrak{J}) \subseteq$ $\operatorname{NHcl}(\operatorname{NHint}(\Theta, \mathfrak{\Im}))$. We know that $\operatorname{NHint}(\Theta$, $\mathfrak{J}) \subseteq(\Theta, \mathfrak{J}) ; \quad$ thus, $\quad \operatorname{NHint}(\Theta, \mathfrak{J}) \subseteq(\Theta, \mathfrak{J}) \subseteq$ $\operatorname{NHcl}(\operatorname{NHint}(\Theta, \mathfrak{\Im})) . \quad \operatorname{Let}(\chi, \mathfrak{B})=\operatorname{NHint}(\Theta, \mathfrak{\Im})$; thus, we get $(\chi, \mathfrak{B}) \subseteq(\Theta, \mathfrak{F}) \subseteq \operatorname{NHcl}(\chi, \mathfrak{B})$.

Conversely, let $(\chi, \mathfrak{B}) \subseteq(\Theta, \mathfrak{J}) \subseteq \operatorname{NHcl}(\chi, \mathfrak{B})$ for some $(\chi, \mathfrak{B}) \in \tau . \quad$ Then, $\quad(\chi, \mathfrak{B}) \subseteq \operatorname{NHint}(\Theta, \mathfrak{J}) \subseteq(\Theta, \mathfrak{F})$. $\Longrightarrow \operatorname{NHcl}(\chi, \mathfrak{B}) \subseteq \operatorname{NHcl}(\operatorname{NHint}(\Theta, \mathfrak{\Im}))$ Thus, $(\Theta, \mathfrak{J}) \subseteq \operatorname{NHcl}(\operatorname{NHint}(\Theta, \mathfrak{J}))$.

Therefore, $(\Theta, \mathfrak{J}) \in \operatorname{NSOHS}(\mathfrak{X})$.

(ii) Let $(\Theta, \mathfrak{J}) \in \operatorname{NSOHS}(\mathfrak{X})$. Then, for some $(\chi, \mathfrak{B}) \in \tau,(\chi, \mathfrak{B}) \subseteq(\Theta, \mathfrak{\Im}) \subseteq \operatorname{NHcl}(\chi, \mathfrak{B})$. If $(\chi, \mathfrak{B}) \subseteq$ $(\Theta, \mathfrak{J}) \subseteq(\xi, \mathfrak{E}), \quad$ then $(\chi, \mathfrak{B}) \subseteq(\xi, \mathfrak{C}) \subseteq \operatorname{NHcl}(\Theta$, $\mathfrak{J}) \subseteq \operatorname{NHcl}(\chi, \mathfrak{B})$. Hence, $(\chi, \mathfrak{B}) \subseteq(\xi, \mathfrak{C}) \subseteq \operatorname{NHcl}(\chi, \mathfrak{B})$. Thus, by $(\mathrm{i}),(\xi, \mathfrak{C}) \in \operatorname{NSOHS}(\mathfrak{X})$.

Definition 17. Let $\left(\mathfrak{X}_{\oplus}, \tau\right)$ be a NHTS and $(\Theta, \mathfrak{J}) \in P(@, \mathfrak{X})$. Then, the largest neutrosophic semiopen hypersoft set contained in $(\Theta, \mathfrak{J})$ is known as the neutrosophic semihypersoft interior of $(\Theta, \mathfrak{\Im})$ and is denoted by FSHSint $(\Theta, \mathfrak{J}), \quad$ i.e., $\quad$ FSHSint $(\Theta, \mathfrak{J})=\cup\{(\chi, \mathfrak{B})$ : $(\chi, \mathfrak{B}) \subseteq(\Theta, \mathfrak{I}),(\chi, \mathfrak{B}) \in \operatorname{FSOHS}(\mathfrak{X})\}$.

And the smallest neutrosophic semiclosed hypersoft set containing $(\Theta, \mathfrak{J})$ is called the neutrosophic semi-hypersoft closure of $(\Theta, \mathfrak{J})$ and is denoted by $\operatorname{NSHScl}(\Theta, \mathfrak{J})$.
$\operatorname{NSHScl}(\Theta, \mathfrak{J})=\cap\{(\xi, \mathfrak{E}):(\xi, \mathfrak{C}) \supseteq(\Theta, \mathfrak{\Im})$ and $(\xi, \mathfrak{E}) \in \operatorname{NSCHS}(\mathfrak{X})\}$

Theorem 25. Let $\left(\mathfrak{X}_{\omega}, \tau\right)$ be a NHTS and $(\Theta, \mathfrak{J})$, $(\chi, \mathfrak{B}) \in P(\emptyset, \mathfrak{X})$. Then, the following properties hold:

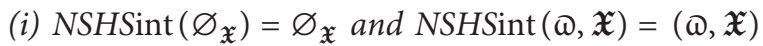

(ii) $\operatorname{NSHSint}(\Theta, \mathfrak{J}) \subseteq(\Theta, \mathfrak{J})$

(iii) NSHSint $(\Theta, \mathfrak{J})$ is the largest neutrosophic semiopen hypersoft set contained in $(\Theta, \mathfrak{J})$

(iv) If $(\Theta, \mathfrak{\Im}) \subseteq(\chi, \mathfrak{B})$, then $\operatorname{NSHSint}(\Theta, \mathfrak{J}) \subseteq$ NSHSint $(\chi, \mathfrak{B})$

(v) $\operatorname{NHSHint}(\operatorname{NHSHint}(\Theta, \mathfrak{J}))=\operatorname{NHSHint}(\Theta, \mathfrak{J})$

(vi) NHSHint $(\Theta, \mathfrak{J}) \cup N H S H i n t(\chi, \mathfrak{B}) \subseteq N H S H i n t[(\Theta$, $\mathfrak{J}) \cup(\chi, \mathfrak{B})]$

(vii) $\operatorname{NHSHint}[(\Theta, \mathfrak{J}) \cap(\chi, \mathfrak{B})] \subseteq N H S H \operatorname{Hint}(\Theta, \mathfrak{J}) \cap$ $\operatorname{NHSHint}(\chi, \mathfrak{B})$

Theorem 26. Let $\left(\mathfrak{X}_{\oplus}, \tau\right)$ be a NHTS and $(\Theta, \mathfrak{J}),(\chi, \mathfrak{B}) \in P(\varpi, \mathfrak{X})$. Then, the following properties hold:

(i) $\operatorname{NSHScl}\left(\varnothing_{\mathfrak{X}}\right)=\varnothing_{\mathfrak{X}}$ and $\operatorname{NSHScl}(@, \mathfrak{X})=(@, \mathfrak{X})$

(ii) $\operatorname{NSHScl}(\Theta, \mathfrak{\Im}) \subseteq(\Theta, \mathfrak{\Im})$

(iii) $\operatorname{NSHScl}(\Theta, \mathfrak{\Im})$ is the minutest neutrosophic semiclosed hypersoft set that holds $(\Theta, \mathfrak{J})$

(iv) If $(\Theta, \mathfrak{\Im}) \subseteq(\chi, B)$, then $\operatorname{NSHScl}(\Theta, \mathfrak{J}) \subseteq$ $\operatorname{NSHScl}(\chi, \mathfrak{B})$

(v) $\operatorname{NHSHcl}(\operatorname{NHSHcl}(\Theta, \mathfrak{\Im}))=\operatorname{NHSHcl}(\Theta, \mathfrak{\Im})$

(vi) $\operatorname{NHSHcl}(\Theta, \mathfrak{\Im}) \cup \operatorname{NHSHcl}(\chi, \mathfrak{B}) \subseteq \mathrm{NHSHcl}$ $[(\Theta, \mathfrak{J}) \cup(\chi, \mathfrak{B})]$

(vii) $\operatorname{NHSHcl}[(\Theta, \mathfrak{\Im}) \cap(\chi, \mathfrak{B})] \subseteq$ $\operatorname{NHSHcl}(\Theta, \mathfrak{J}) \cap \operatorname{NHSHcl}(\chi, \mathfrak{B})$

Theorem 27. Every neutrosophic open (closed) hypersoft set in a NHTS $\left(\mathfrak{X}_{\oplus}, \tau\right)$ is a neutrosophic semiopen (closed) hypersoft set.

Proof. Let $(\Theta, \mathfrak{J})$ be a neutrosophic open hypersoft set. Then, $\operatorname{NHint}(\Theta, \mathfrak{J})=(\Theta, \mathfrak{J})$. Since $(\Theta, \mathfrak{J}) \subseteq \operatorname{NHcl}(\Theta, \mathfrak{J})$, $(\Theta, \mathfrak{J}) \subseteq \operatorname{NHcl}(\operatorname{NHint}(\Theta, \mathfrak{J}))$. Thus, $(\Theta, \mathfrak{J}) \in \mathrm{NSOHS}$ $(\mathfrak{X})$.

Theorem 28. Let $\left(\mathfrak{X}_{\oplus}, \tau\right)$ be a NHTS and $(\Theta, \mathfrak{J}),(\chi, \mathfrak{B}) \in P(\emptyset, \mathfrak{X})$. If either $(\Theta, \mathfrak{J}) \in \operatorname{NSOHS}(X)$ or $(\chi, \mathfrak{B}) \in \operatorname{NSOHS}(\mathfrak{X})$, then $\operatorname{NHcl}(\operatorname{NHint}(\Theta, \mathfrak{J}) \cap(\chi, \mathfrak{B}))=$ $\operatorname{NHcl}(N H$ int $(\Theta, \mathfrak{\Im}) \cap \operatorname{NHclNHint}(\chi, \mathfrak{B}))$.

Proof. Let $(\Theta, \mathfrak{\Im}),(\chi, \mathfrak{B}) \in P(\mathfrak{⿴}, \mathfrak{X})$. Then, we have 


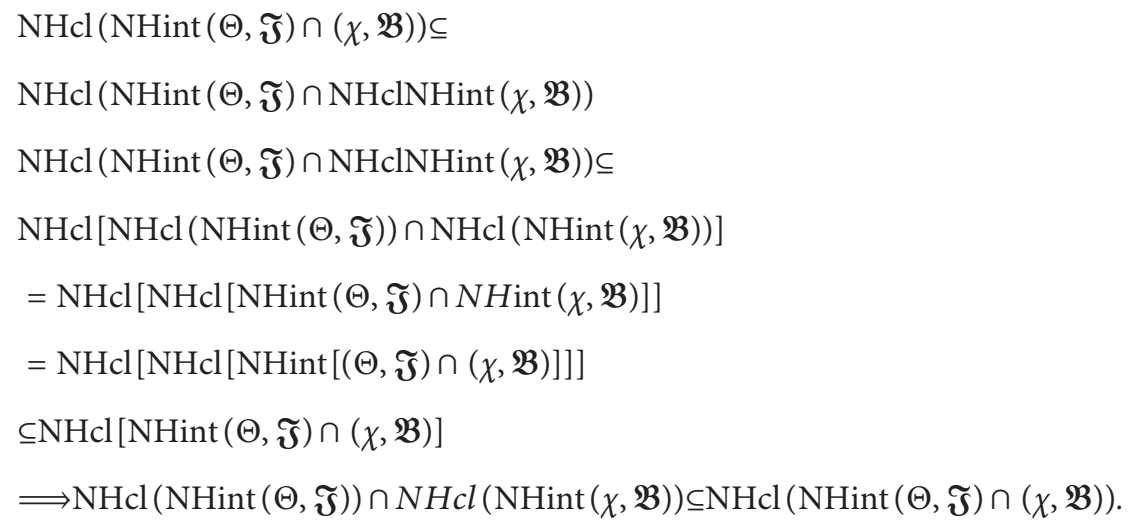

Thus, $\quad \operatorname{NHcl}(\operatorname{NHint}(\Theta, \mathfrak{S}) \cap(\chi, \mathfrak{B}))=\quad$ NHclNHint $(\Theta, \mathfrak{J}) \cap \operatorname{NHcl}(\operatorname{NHint}(\chi, \mathfrak{B}))$.

Theorem 29. Let $\left(\mathfrak{X}_{\omega}, \tau\right)$ be a NHTS, $(\Theta, \mathfrak{J})$ be a neutrosophic hypersoft open set, and $(\chi, \mathfrak{B}) \in \operatorname{NSOHS}(\mathfrak{X})$. Then, $(\Theta, \mathfrak{J}) \cap(\chi, \mathfrak{B}) \in \operatorname{NSOHS}(\mathfrak{X})$.

Proof. Let $(\Theta, \mathfrak{I})$ be a NOHS set and $(\chi, \mathfrak{B})$ be a NSOHS. Then, $\quad(\Theta, \mathfrak{I}) \cap(\chi, \mathfrak{B}) \supseteq N H i n t((\Theta, \mathfrak{I}) \cap(\chi, \mathfrak{B})) \Longrightarrow$ NHint $((\Theta, \mathfrak{J}) \cap(\chi, \mathfrak{B})) \subseteq(\Theta, \mathfrak{J}) \cap(\chi, \mathfrak{B})$. Then, $\operatorname{NHint}((\Theta$, $\mathfrak{\Im}) \cap(\chi, \mathfrak{B})) \subseteq(\Theta, \mathfrak{I}) \cap(\chi, \mathfrak{B}) \subseteq \operatorname{NHclNHint}((\Theta, \quad \mathfrak{\Im}) \cap(\chi$, $\mathfrak{B})) . \Longrightarrow(\Theta, \mathfrak{I}) \cap(\chi, \mathfrak{B}) \subseteq \operatorname{NHclNHint}((\Theta, \mathfrak{\Im}) \cap(\chi, \mathfrak{B}))$.

Therefore, $(\Theta, \mathfrak{I}) \cap(\chi, \mathfrak{B})$ is a NSOHS.

Proposition 5. Let $(\Theta, \mathfrak{J})$ be a neutrosophic hypersoft set in the NHTS $\left(\mathfrak{X}_{\oplus}, \tau\right)$. Then, $(\Theta, \mathfrak{J})$ is NSCHS if and only if there exists a NCHS set $(\xi, \mathfrak{\Im})$ such that $\operatorname{NHint}(\xi$, $\mathfrak{\Im}) \subseteq(\Theta, \mathfrak{J}) \subseteq(\xi, \mathfrak{\Im})$

Proposition 6. Every neutrosophic hypersoft closed set is a NSCHS set in a NHTS $\left(\mathfrak{X}_{\mathfrak{W}}, \tau\right)$, but the converse need not be true.

Theorem 30. Let $(\Theta, \mathfrak{J})$ be a NHS in a NHTS $\left(\mathfrak{X}_{\oplus}, \tau\right)$. Then, $(\Theta, \mathfrak{J})$ is NSCHS if and only if $\operatorname{NHint}(\operatorname{NHcl}(\Theta, \mathfrak{J})) \subseteq(\Theta, \mathfrak{J})$.

Proof. Suppose $(\Theta, \mathfrak{\Im})$ is a NSCHS set; then, there exists a $\operatorname{NHCS}(\Theta, \mathfrak{J})$ such that $\operatorname{NHint}(\xi, \mathfrak{J}) \subseteq(\Theta, \mathfrak{J}) \subseteq(\xi, \mathfrak{J})$. $\operatorname{NHcl}(\Theta, \mathfrak{I}) \subseteq \operatorname{NHcl}(\xi, \mathfrak{I})=(\xi, \mathfrak{\Im})$.

Thus, $\operatorname{NHint}(\operatorname{NHcl}(\Theta, \mathfrak{J})) \subseteq \operatorname{NHint}(\xi, \quad \mathfrak{I}) \subseteq(\Theta, \mathfrak{J}) \Longrightarrow$ $\operatorname{NHint}(\operatorname{NHcl}(\xi, \mathfrak{\Im})) \subseteq(\Theta, \mathfrak{J})$.

Conversely, let $(\Theta, \mathfrak{\Im})$ be a neutrosophic hypersoft set in $\left(\mathfrak{X}_{\varpi}, \tau\right)$ such that $\operatorname{NHint}(\operatorname{NHcl}(\Theta, \mathfrak{J})) \subseteq(\Theta, \mathfrak{J})$.

Let $\operatorname{NHcl}(\Theta, \mathfrak{\Im})=(\xi, \mathfrak{\Im})$.

Then, $\operatorname{NHint}(\Theta, \mathfrak{J}) \subseteq(\Theta, \mathfrak{J}) \subseteq(\xi, \mathfrak{J})$.

Thus, $(\Theta, \mathfrak{J})$ is a NSCHS.

Theorem 31. Let $\left\{(\Theta, \mathfrak{\Im})_{\beta}: \beta \in I\right\}$ be a family of NSCHSs in a NHTS $\left(\mathfrak{X}_{\varrho}, \tau\right)$. Then, the intersection $\cap_{\beta \in I}(\Theta, \mathfrak{J})_{\beta}$ is a NSCHS in $\left(\mathfrak{X}_{\varpi}, \tau\right)$.

Proof. Since each $\beta \in I,(\Theta, \mathfrak{J})_{\beta}$ is a NSCHS.

Then, there exists a NCHS $(\xi, \mathfrak{J})_{\beta}$ such that $\operatorname{NHint}\left((h, A)_{\beta}\right) \subseteq(\Theta, \mathfrak{J})_{\beta} \subseteq(\xi, \mathfrak{J})_{\beta}$.
Thus, $\quad \cap_{\beta \in I}\left(\operatorname{NHint}\left((\xi, \mathfrak{J})_{\beta}\right)\right) \subseteq \cap_{\beta \in I}(\Theta, \mathfrak{J})_{\beta} \quad \cap_{\beta \in I}$ (NHint $\left.\left((\xi, \mathfrak{J})_{\beta}\right)\right) \subseteq \cap_{\beta \in I}(\Theta, \mathfrak{J})_{\beta} \subseteq \cap_{\beta \in \mathrm{I}}(\xi, \mathfrak{J})_{\beta}$. Consider $\cap_{\beta \in I}(\xi, \mathfrak{J})_{\beta}=(\xi, \mathfrak{J})$.

Then, $(\xi, \mathfrak{J})$ is a NCHS, and hence, $\cap_{\beta \in I}(\Theta, \mathfrak{J})_{\beta}$ is a NSCHS.

Theorem 32. Let $(\Theta, \mathfrak{\Im})$ be a NSCHS and $(\vartheta, \mathfrak{\Im})$ be a NCHS in $\left(\mathfrak{X}_{\Theta}, \tau\right)$. If $\operatorname{NHint}(\Theta, \mathfrak{J}) \subseteq(\vartheta, \mathfrak{J}) \subseteq(\Theta, \mathfrak{J})$, then $(\vartheta, \mathfrak{J})$ is a NSCHS.

Proof. Since $(\Theta, \mathfrak{J})$ is a NSCHS, there exists a NCHS $(\xi, \mathfrak{J})$ such that $\operatorname{NHint}(\xi, \mathfrak{J}) \subseteq(\Theta, \mathfrak{J}) \subseteq(\xi, \mathfrak{J})$. Then, $(\vartheta, \mathfrak{J}) \subseteq(\xi, \mathfrak{J})$. Also, $\operatorname{NHintFHint}(\xi, \mathfrak{I}) \subseteq \operatorname{NHint}(\xi, \mathfrak{\Im}) \subseteq \operatorname{NHint}(\Theta, \mathfrak{\Im})$. $\Longrightarrow$ $\operatorname{NHint}(\xi, \mathfrak{J}) \subseteq(\vartheta, \mathfrak{J})$. Therefore, $\operatorname{NHint}(\xi, \mathfrak{J}) \subseteq(\vartheta, \mathfrak{J}) \subseteq$ $(\xi, \mathfrak{\Im})$.

Hence, $(\Theta, \mathfrak{J})$ is a NSCHS.

Remark 5. For any NCHS $(\Theta, \mathfrak{J}), \operatorname{NSHScl}(\Theta, \mathfrak{J})=(\Theta, \mathfrak{J})$. And for any NOHS $(\zeta, \mathfrak{J})$, NSHSint $(\zeta, \mathfrak{J})=(\zeta, \mathfrak{J})$.

Remark 6. If $(\Theta, \mathfrak{\Im})$ is a neutrosophic hypersoft set in $\left(\mathfrak{X}_{\mathscr{\omega}}, \tau\right)$, then $\operatorname{NHint}(\Theta, \mathfrak{J}) \subseteq \operatorname{NSHSint}(\Theta, \mathfrak{J}) \subseteq(\Theta, \mathfrak{J}) \subseteq$ $\operatorname{NSHScl}(\Theta, \mathfrak{J}) \subseteq \operatorname{NHcl}(\Theta, \mathfrak{J})$.

Theorem 33. Let $(\Theta, \mathfrak{J})$ be a NHS in $\left(\mathfrak{X}_{\oplus}, \tau\right)$. Then,

(i) $(\operatorname{NSHSint}(\Theta, \mathfrak{\Im}))^{C}=\operatorname{NSHScl}\left((\Theta, \mathfrak{\Im})^{C}\right)$

(ii) $(\operatorname{NSHScl}(\Theta, \mathfrak{\Im}))^{C}=\operatorname{NSHSint}\left((\Theta, \mathfrak{\Im})^{C}\right)$

(iii) $\operatorname{NSHSint}(F H i n t(\Theta, \mathfrak{\Im})) \backslash \backslash=N H$ int $(F \operatorname{FHSint}(\Theta, \mathfrak{J}))=\operatorname{NHint}(\Theta, \mathfrak{\Im})$

(iv) $\operatorname{NSHScl}(\operatorname{FHcl}(\Theta, \mathfrak{\Im}))=\operatorname{NHcl}(\operatorname{FSHScl}(\Theta, \mathfrak{\Im})) \backslash \backslash$ $=\operatorname{NHcl}(\Theta, \mathfrak{\Im})$

Proof

(i) $\operatorname{NSHSint}(\Theta, \mathfrak{J}) \subseteq(\Theta, \mathfrak{\Im}) \Longrightarrow(\Theta, \mathfrak{I})^{C} \subseteq(\operatorname{NSHSint}(\Theta$, $\mathfrak{\Im}))^{C}$.

Since $(\operatorname{NSHSint}(\Theta, \mathfrak{J}))^{C}$ is a NSCHS, NSHScl $(\Theta, \mathfrak{\Im})^{C} \subseteq \operatorname{NSHScl}\left((\operatorname{NSHSint}(\Theta, \mathfrak{J}))^{C}\right)=$ (NSHS int $(\Theta, \mathfrak{J}))^{C}$. 
Conversely, $(\Theta, \mathfrak{T})^{C} \subseteq \mathrm{NSHScl}\left((\Theta, \mathfrak{T})^{C}\right) \Longrightarrow \mathrm{NSHScl}$ $\left((\Theta, \mathfrak{J})^{C}\right)^{C} \subseteq\left((\Theta, \mathfrak{J})^{C}\right)^{C}=(\Theta, \mathfrak{J})$.

$\operatorname{NSHScl}\left((\Theta, \mathfrak{J})^{C}\right)$ being FSCHS implies that $\operatorname{NSHScl}\left((\Theta, \mathfrak{\Im})^{C}\right)^{C}$ is a FSOHS.

Thus, $\operatorname{NSHScl}\left((\Theta, \mathfrak{J})^{C}\right)^{C} \subseteq \operatorname{NSHSint}(\Theta, \mathfrak{J})$.

And hence, $(\mathrm{NSHSint}(\Theta, \mathfrak{J}))^{C} \subseteq(\mathrm{NSHScl}$ $\left.\left((\Theta, \mathfrak{J})^{C}\right)^{C}\right)^{C}=\left(\operatorname{NSHScl}\left((\Theta, \mathfrak{J})^{C}\right)\right)$.

(ii) The proof is the same as that of (i).

(iii) $\operatorname{NHint}(\Theta, \mathfrak{J})$ is FOHS implying that it is FSOHS.

Therefore, $\operatorname{NSHSint}(\operatorname{NHint}(\Theta, \mathfrak{J}))=\operatorname{NHint}(\Theta, \mathfrak{\Im})$.

$\operatorname{Now}, \operatorname{NHint}(\Theta, \mathfrak{J}) \subseteq \operatorname{NSHSint}(\Theta, \mathfrak{J})=(\Theta, \mathfrak{J})$. Thus, $\operatorname{NSHSint}(\operatorname{NHint}(\Theta, \mathfrak{J}))=\operatorname{NHint}(\Theta, \mathfrak{J})$.

(iv) $\operatorname{NHcl}(\Theta, \mathfrak{J})$ is neutrosophic closed hypersoft implying that it is NSCHS.

Therefore, $\operatorname{NSHScl}(\operatorname{NHcl}(\Theta, \mathfrak{J}))=\operatorname{NHcl}(\Theta, \mathfrak{\Im})$. Now, $(\Theta, \mathfrak{J}) \subseteq \operatorname{NSHScl}(\Theta, \mathfrak{J}) \subseteq \operatorname{NHcl}(\Theta, \mathfrak{J})$.

Hence, $\quad \operatorname{NSHScl}(\Theta, \mathfrak{J}) \subseteq \operatorname{NHclNSHScl}((\Theta, \mathfrak{J})) \backslash \backslash \subseteq$ $\operatorname{NSHScl}(\Theta, \mathfrak{J})$.

This implies $\operatorname{NHcl}(\operatorname{NSHScl}(\Theta, \mathfrak{J})) \subseteq \operatorname{NHcl}(\Theta, \mathfrak{J})$.

\section{Application}

In this section, we present a multiattribute group decisionmaking (MAGDM) application of the NHS and NHS topology using two different algorithms, and the results of both algorithms are compared at the end. The algorithms proposed in [36] are considered, and some of their techniques are followed. Hypersoft sets are more feasible than soft sets and are more advantageous to use for applications since they can be dealt with more uncertainties. There are many methods proposed for multiattribute group decisionmaking applications, but the proposed method is feasible than the methods which were proposed beforehand and done by using the more advanced recent work.

6.1. Numerical Example. We propose to analyse the risk of COVID-19 by two MAGDM methods described by Algorithms 1 and 2 based on neutrosophic hypersoft sets and topology. We have all been affected by the current COVID19 pandemic. However, the impact and consequences of the pandemic vary depending on our status as individuals and members of the society. We all find it difficult to be treated in hospitals because COVID affects everyone regardless of age. As a result, determining who should be treated first and assisting the most affected in becoming cured are difficult. The following method proposes methods for reducing the risk and treating patients based on their high risk of virus infection. Suppose that a committee of doctors have to give a report on patients having risk of COVID-19 in a particular area or hospital.

Let $X=\left\{p_{1}, p_{2}, p_{3}, p_{4}, p_{5}\right\}$ be the patients reported to the hospital. Suppose that the doctors consider the following set of attributes: $E=\left\{e_{1}, e_{2}, e_{3}, e_{4}, e_{5}\right\}$, where the attributes are $e_{1}=$ age, $e_{2}=$ illness, and $e_{3}=$ symptoms of the patients.
The attributes are subclassified as $E_{1}=\left\{e_{11}, e_{12}, e_{13}\right.$, $\left.e_{14}, e_{15}\right\}=$ age, where $e 11$ is people of age 0 to $17, e 12$ is people of age 18 to $44, e 13$ is people of age 45 to $64, e 14$ is people of age 65 to 74 , and $e 15$ is people of age $75+$.

$E_{2}=\left\{e_{21}, e_{22}, e_{23}, e_{24}\right\}=$ illness, where $e_{21}, e 22, e_{23}$, and $e_{24}$ represent the patients with diabetes and hypertension, cardiovascular disease, chronic respiratory disease, and cancer, respectively.

$E_{3}=\left\{e_{31}, e_{32}, e_{33}\right\}=$ immune level, where e31,e32, and e33 represent people with low, medium, and high level of immune count.

$E_{4}=\left\{e_{41}, e_{42}, e_{43}\right\}=$ symptoms, where $e_{41}$ is the person having most common symptoms (fever, dry cough, and tiredness), $e_{42}$ is the person having less common symptoms (aches and pain, sore throat, diarrhoea, headache, and loss of taste or smell), and $e_{43}$ is the person having serious symptoms (shortness of breath, chest pain, and loss of speech or movement).

Doctors divide the criteria into two subsets, $\mathfrak{A}$ (category 1 , for higher risk) and $\mathfrak{B}$ (category 2 , for medium risk).

Category 1: $\mathfrak{A}$ represents attributes $e_{3}$ and $e_{4}$

Category 2: $\mathfrak{B}$ represents attributes $e_{2}, e_{3}$, and $e_{4}$

First, we solve the problem by using the NHS-MAGDM method as described in Algorithm 1.

Step 1: two NHSs, namely, $(f, \mathfrak{A})$ and $(g, \mathfrak{B})$ over $X$, are constructed after receiving all the required data from the committee.

$(f, \mathfrak{A})=\left\{\alpha_{1}, \alpha_{2}, \alpha_{3}\right\}, \quad$ where $\quad \alpha_{1}=f\left(e_{31}, e_{43}\right), \alpha_{2}=$ $f\left(e_{32}, e_{43}\right)$, and $\alpha_{3}=f\left(e_{31}, e_{42}\right)$, and $(g, \mathfrak{B})=\left\{\beta_{1}, \beta_{2}\right.$, $\left.\beta_{3}\right\}$, where $\beta_{1}=g\left(e_{21}, e_{31}, e_{43}\right), \beta_{2}=g\left(e_{21}, e_{32}, e_{42}\right)$, and $\beta_{3}=g\left(e_{22}, e_{32}, e_{41}\right)$. The values for the NHS $(f, \mathfrak{A})$ and $(g, \mathfrak{B})$ are given in Tables 1 and 2.

Step 2: we are now constructing the NHS topology given by $\tau=\{\varnothing, X,(f, \mathfrak{A}),(g, \mathfrak{B})\}$, where $\varnothing, X$ are NHS empty and full sets. The neutrosophic hypersoft open set $(f, \mathfrak{A})$ and $(g, \mathfrak{B})$ are formed in Tables 3 and 4 , respectively, by taking the average for each element from Tables 1 and 2 .

Step 3: the score matrix of NHS sets $(f, \mathfrak{A})$ and $(g, \mathfrak{B})$ is calculated in Tables 5 and 6, respectively.

Step 4: we are now calculating the decision table of $(f, \mathfrak{A})$ and $(g, \mathfrak{B})$ by averaging the score values correspondingly. Table 7 gives the decision values of $(f, \mathfrak{A})$ and $(g, \mathfrak{B})$.

Step 5: now, by adding the decision values of $(f, \mathfrak{A})$ and $(g, \mathfrak{B})$, we find the final decision value. Table 8 is the required final decision table.

Step 6: using Table 8, the final ranking of the patients is given by

$$
p_{2}>p_{3}>p_{5}>p_{1}>p_{4}
$$

We see that patient 2 has the maximum value. So, patient 2 is selected for the immediate treatment. 
Step 1: input $\operatorname{NHS}(f, \mathfrak{A})$ and $(g, \mathfrak{B})$.

Step 2: construct NHS topology $\tau$ such that $(f, \mathfrak{A})$ and $(g, \mathfrak{B})$ are ONHS in $\tau$. Construct the hypersoft open set $(f, \mathfrak{A})$ such that, for each element, the average is taken to form the table.

Step 3: calculate the score matrix corresponding to each ONHS. ( $f, \mathfrak{A})$ denotes the neutrosophic hypersoft set; then, the neutrosophic set $\left(f_{T}, \mathfrak{A}\right)$ in which each entry in the set $f_{T}(e)$ is the score function of the respective entries in the hypersoft set $f(e)$ is called the score matrix. For each hypersoft element $f(x), s(f)=(1 / n(f)) \sum_{\varphi \in f(x)} \varphi$ is the score function of $f(x)$, where $n(f)$ is the number of values in $f(x)$.

Step 4: calculate the average of each ONHS for each $p_{i}$, and let it be denoted by $d_{i}$ and $e^{i}$. This is the decision table for each ONHS. Step 5: add the decision table of ONHS $(f, \mathfrak{A})$ and $(g, \mathfrak{B})$. This is the final decision table.

Step 6: select the optimal alternative $p_{i}$ using $\max \left\{d_{i}+e_{i}\right\}$.

Algorithm 1:

Step 1: input NHS $(f, \mathfrak{U})$ and $(g, \mathfrak{B})$.

Step 2: construct NHS topology $\tau$ such that $(f, \mathfrak{A})$ and $(g, \mathfrak{B})$ are ONHS in $\tau$. Construct the hypersoft open set $(f, \mathfrak{A})$ such that, for each element, the average is taken to form the table.

Step 3: calculate the score matrix corresponding to each ONHS. $(f, \mathfrak{A})$ denotes the neutrosophic hypersoft set; then, the neutrosophic set $\left(f_{T}, \mathfrak{A}\right)$ in which each entry in the set $f_{T}(e)$ is the score function of the respective entries in the hypersoft set $f(e)$ is called the score matrix. For each hypersoft element $f(x), s(f)=(1 / n(f)) \sum_{\varphi \in f(x)} \varphi$ is the score function of $f(x)$, where $n(f)$ is the number of values in $f(x)$.

Step 4: find the cardinality of all ONHSs by using $C(f, \mathfrak{A})=\{C f(a) / a: a \in \mathfrak{A}\}$, where $C f(a)=\sum_{p \in X} f(p) /\langle X\rangle$.

Step 5: find the aggregate fuzzy set of the score matrix by using $|E|^{*} M_{(f, \mathfrak{A})^{*}}=M_{(f, \mathfrak{A})} * M_{C(f, \mathfrak{A})}^{t}$, where $M_{(f, \mathfrak{A})^{*}}, M_{(f, \mathfrak{A})}$, and $M_{C(f, \mathfrak{A})}^{t}$ represent the aggregate fuzzy matrix, score matrix, and transpose of the cardinal set, respectively.

Step 6: add $(f, \mathfrak{A})^{*}$ and $(g, \mathfrak{B})^{*}$ to find decision NHS.

Step 7: determine the optimal choice given by $\max \left\{(f, \mathfrak{A})^{*}+(g, \mathfrak{B})^{*}(p)\right\}$.

Algorithm 2:

Table 1: Values for $(f, \mathfrak{A})$.

\begin{tabular}{|c|c|c|c|c|c|c|}
\hline$(f, A)$ & & & & & & \\
\hline$X$ & $e_{31}$ & $e_{43}$ & $e_{32}$ & $e_{43}$ & $e_{31}$ & $e_{42}$ \\
\hline$p_{1}$ & $(0.5,0.4,0.1)$ & $(0.1,0.1,0.1)$ & $(0.5,0.3,0.2)$ & $(0.1,0.1,0.1)$ & $(0.5,0.4,0.1)$ & $(0.8,0.2,0.1)$ \\
\hline$p_{2}$ & $(0.7,0.2,0.2)$ & $(0.7,0.2,0.1)$ & $(0.6,0.4,0.2)$ & $(0.7,0.2,0.1)$ & $(0.7,0.2,0.2)$ & $(0.6,0.1,0.2)$ \\
\hline$p_{3}$ & $(0.1,0.4,0.4)$ & $(0.8,0.4,0.2)$ & $(0.8,0.1,0.1)$ & $(0.8,0.4,0.2)$ & $(0.1,0.4,0.4)$ & $(0.5,0.2,0.1)$ \\
\hline$p_{4}$ & $(0.1,0.4,0.1)$ & $(0.5,0.2,0.1)$ & $(0.1,0.2,0.1)$ & $(0.5,0.2,0.1)$ & $(0.1,0.4,0.1)$ & $(0.3,0.2,0.1)$ \\
\hline$p_{5}$ & $(0.8,0.3,0.1)$ & $(0.6,0.4,0.2)$ & $(0.6,0.2,0.1)$ & $(0.6,0.4,0.2)$ & $(0.8,0.3,0.1)$ & $(0.4,0.1,0.1)$ \\
\hline
\end{tabular}

TABle 2: Values for $(g, \mathfrak{B})$.

\begin{tabular}{ccccccccccc}
\hline$X$ & \multicolumn{3}{c}{$\beta_{1}$} & \multicolumn{8}{c}{$\beta_{2}$} & & & \multicolumn{3}{c}{$\beta_{3}$} \\
& $e_{21}$ & $e_{31}$ & $e_{43}$ & $e_{21}$ & $e_{32}$ & $e_{42}$ & $e_{22}$ & $e_{32}$ & $e_{41}$ \\
\hline$p_{1}$ & $(0.8,0.2,0.1)$ & $(0.5,0.4,0.1)$ & $(0.1,0.1,0.1)$ & $(0.8,0.2,0.1)$ & $(0.5,0.3,0.2)$ & $(0.8,0.2,0.1)$ & $(0.4,0.2,0.2)$ & $(0.5,0.3,0.2)$ & $(0.9,0.1,0.1)$ \\
$p_{2}$ & $(0.9,0.2,0.1)$ & $(0.7,0.2,0.2)$ & $(0.7,0.2,0.1)$ & $(0.9,0.2,0.1)$ & $(0.6,0.4,0.2)$ & $(0.6,0.1,0.2)$ & $(0.6,0.3,0.2)$ & $(0.6,0.4,0.2)$ & $(0.8,0.2,0.2)$ \\
$p_{3}$ & $(0.5,0.3,0.4)$ & $(0.1,0.4,0.4)$ & $(0.8,0.4,0.2)$ & $(0.5,0.3,0.4)$ & $(0.8,0.1,0.1)$ & $(0.5,0.2,0.1)$ & $(0.8,0.2,0.2)$ & $(0.8,0.1,0.1)$ & $(0.6,0.4,0.2)$ \\
$p_{4}$ & $(0.6,0.4,0.2)$ & $(0.1,0.4,0.1)$ & $(0.5,0.2,0.1)$ & $(0.6,0.4,0.2)$ & $(0.1,0.2,0.1)$ & $(0.3,0.2,0.1)$ & $(0.7,0.3,0.2)$ & $(0.1,0.2,0.1)$ & $(0.5,0.3,0.2)$ \\
$p_{5}$ & $(0.3,0.2,0.1)$ & $(0.8,0.3,0.1)$ & $(0.6,0.4,0.2)$ & $(0.3,0.2,0.1)$ & $(0.6,0.2,0.1)$ & $(0.4,0.1,0.1)$ & $(0.4,0.2,0.1)$ & $(0.6,0.2,0.1)$ & $(0.6,0.3,0.2)$ \\
\hline
\end{tabular}

TABLE 3: The tabular representation of $(f, \mathfrak{A})$.

\begin{tabular}{lccc}
\hline$X$ & $\alpha_{1}$ & $\alpha_{2}$ & $\alpha_{3}$ \\
\hline$p_{1}$ & $(0.3,0.25,0.1)$ & $(0.3,0.2,0.15)$ & $(0.65,0.30,0.1)$ \\
$p_{2}$ & $(0.7,0.2,0.15)$ & $(0.65,0.3,0.15)$ & $(0.65,0.15,0.2)$ \\
$p_{3}$ & $(0.45,0.4,0.3)$ & $(0.8,0.2,0,1)$ & $(0.3,0.3,0.25)$ \\
$p_{4}$ & $(0.3,0.3,0.1)$ & $(0.3,0.2,0.1)$ & $(0.2,0.3,0.1)$ \\
$p_{5}$ & $(0.7,0.35,0.15)$ & $(0.6,0.3,0.15)$ & $(0.6,0.2,0.1)$ \\
\hline
\end{tabular}


TABLE 4: The tabular representation of $(g, \mathfrak{B})$.

\begin{tabular}{cccc}
\hline$X$ & $\beta_{1}$ & $\beta_{2}$ & $\beta_{3}$ \\
\hline$p_{1}$ & $(0.47,0.23,0.1)$ & $(0.7,0.23,0.13)$ & $(0.6,0.2,0.17)$ \\
$p_{2}$ & $(0.77,0.2,0.13)$ & $(0.7,0.23,0.17)$ & $(0.67,0.3,0.2)$ \\
$p_{3}$ & $(0.47,0.37,0.33)$ & $(0.6,0.2,0.2)$ & $(0.73,0.23,0.17)$ \\
$p_{4}$ & $(0.4,0.33,0.13)$ & $(0.33,0.27,0.13)$ & $(0.43,0.27,0.17)$ \\
$p_{5}$ & $(0.57,0.3,0.13)$ & $(0.43,0.17,0.1)$ & $(0.53,0.23,0.13)$ \\
\hline
\end{tabular}

TABLe 5: Score matrix of $(f, \mathfrak{U})$.

\begin{tabular}{cccc}
\hline$X$ & $\alpha_{1}$ & $\alpha_{2}$ & $\alpha_{3}$ \\
\hline$p_{1}$ & 0.217 & 0.217 & 0.35 \\
$p_{2}$ & 0.35 & 0.37 & 0.33 \\
$p_{3}$ & 0.38 & 0.37 & 0.28 \\
$p_{4}$ & 0.23 & 0.2 & 0.2 \\
$p_{5}$ & 0.4 & 0.35 & 0.3 \\
\hline
\end{tabular}

TABLE 6: Score matrix of $(g, \mathfrak{B})$.

\begin{tabular}{lccc}
\hline$X$ & $\beta_{1}$ & $\beta_{2}$ & $\beta_{3}$ \\
\hline$p_{1}$ & 0.27 & 0.35 & 0.32 \\
$p_{2}$ & 0.37 & 0.37 & 0.39 \\
$p_{3}$ & 0.39 & 0.33 & 0.38 \\
$p_{4}$ & 0.29 & 0.24 & 0.29 \\
$p_{5}$ & 0.33 & 0.23 & 0.29 \\
\hline
\end{tabular}

Table 7: Decision table.

\begin{tabular}{lccc}
\hline$(f, \mathfrak{A})$ & & \multicolumn{2}{c}{$(g, \mathfrak{B})$} \\
\hline$d_{i}$ & Values & $e_{i}$ & Values \\
$d_{1}$ & 0.261 & $e_{1}$ & 0.313 \\
$d_{2}$ & 0.35 & $e_{2}$ & 0.376 \\
$d_{3}$ & 0.34 & $e_{3}$ & 0.367 \\
$d_{4}$ & 0.21 & $e_{4}$ & 0.273 \\
$d_{5}$ & 0.35 & $e_{5}$ & 0.283 \\
\hline
\end{tabular}

TABle 8: Final decision table.

\begin{tabular}{lc}
\hline$d_{i}+e_{i}$ & Values \\
\hline$d_{1}+e_{1}$ & 0.574 \\
$d_{2}+e_{2}$ & 0.726 \\
$d_{3}+e_{3}$ & 0.707 \\
$d_{4}+e_{4}$ & 0.483 \\
$d_{5}+e_{5}$ & 0.633 \\
\hline
\end{tabular}

Using Algorithm 2, we are now solving the same problem.

Steps 1, 2, and 3 are identical to those in Algorithm 1. Step 4: the cardinal is computed by the formula given above in the algorithm. The cardinal for $(f, \mathfrak{A})$ is

$$
C(f, \mathfrak{A})=\{0.315,0.301,0.292\} .
$$

Similarly, the cardinal for $(g, \mathfrak{B})$ is

$$
C(g, \mathfrak{B})=\{0.33,0.304,0.334\}
$$

and the cardinal for empty and full sets is completely 0 and 1 , respectively.

Step 5: we are now finding the fuzzy matrix aggregate $M_{(f, \mathfrak{A})^{*}}$ :

$$
M_{(f, \mathfrak{A})^{*}}=\frac{1}{4}\left[\begin{array}{ccc}
0.217 & 0.217 & 0.35 \\
0.35 & 0.37 & 0.33 \\
0.38 & 0.37 & 0.28 \\
0.23 & 0.2 & 0.2 \\
0.4 & 0.35 & 0.3
\end{array}\right]\left[\begin{array}{l}
0.315 \\
0.301 \\
0.292
\end{array}\right]
$$

$$
=\frac{1}{4}\left[\begin{array}{l}
0.236 \\
0.318 \\
0.313 \\
0.191 \\
0.319
\end{array}\right]=\left[\begin{array}{l}
0.059 \\
0.0795 \\
0.07825 \\
0.04775 \\
0.07975
\end{array}\right] .
$$

Thus, we obtain aggregate fuzzy set $(f, \mathfrak{A})^{*}$ given by $(f, \mathfrak{U})^{*}=\left\{\left(p_{1}, 0.059\right),\left(p_{2}, 0.0795\right),\left(p_{3}, 0.07825\right),\left(p_{4}\right.\right.$, $\left.0.04775),\left(p_{5}, 0.07975\right)\right\}$.

We can also find an aggregate fuzzy matrix, $M_{(g, \mathfrak{B})^{*}}$ :

$M_{(g, \mathfrak{B})^{*}}=\frac{1}{4}\left[\begin{array}{ccc}0.27 & 0.35 & 0.32 \\ 0.37 & 0.37 & 0.39 \\ 0.39 & 0.33 & 0.38 \\ 0.29 & 0.24 & 0.29 \\ 0.33 & 0.23 & 0.29\end{array}\right]\left[\begin{array}{l}0.33 \\ 0.304 \\ 0.334\end{array}\right]$

$$
=\frac{1}{4}\left[\begin{array}{l}
0.3525 \\
0.3649 \\
0.356 \\
0.2657 \\
0.2758
\end{array}\right]=\left[\begin{array}{l}
0.076 \\
0.091 \\
0.089 \\
0.066 \\
0.0689
\end{array}\right],
$$

$$
\begin{aligned}
(g, \mathfrak{B})^{*}= & \left\{\left(p_{1}, 0.076\right),\left(p_{2}, 0.091\right),\left(p_{3}, 0.089\right)\right. \\
& \left.\left(p_{4}, 0.066\right),\left(p_{5}, 0.0689\right)\right\}
\end{aligned}
$$

Step 6: now, by adding the aggregate fuzzy sets, we find the final decision set $(f, \mathfrak{A})^{*}$ and $(g, \mathfrak{B})^{*}$ : 
TABLE 9: Comparison of the final ranking obtained by both algorithms.

\begin{tabular}{lcc}
\hline Method & Ranking & Optimal decision \\
\hline Algorithm 1 & $p_{2}>p_{3}>p_{5}>p_{1}>p_{4}$ & $p_{2}$ \\
Algorithm 2 & $p_{2}>p_{3}>p_{5}>p_{1}>p_{4}$ & $p_{2}$ \\
\hline
\end{tabular}

$$
\begin{aligned}
& (f, \mathfrak{A})^{*}(p)=\left\{\left(p_{1}, 0.059\right),\left(p_{2}, 0.0795\right),\left(p_{3}, 0.07825\right),\left(p_{4}, 0.04775\right),\left(p_{5}, 0.07975\right)\right\} \\
& (g, \mathfrak{B})^{*}(p)=\left\{\left(p_{1}, 0.076\right),\left(p_{2}, 0.091\right),\left(p_{3}, 0.089\right),\left(p_{4}, 0.066\right),\left(p_{5}, 0.0689\right)\right\} .
\end{aligned}
$$

Thus, $\quad\left\{(f, \mathfrak{A})^{*}(p)+(g, \mathfrak{B})^{*}(p)\right\}=\left\{\left(p_{1}, 0.135\right)\right.$ $\left.\left(p_{2}, 0.170\right),\left(p_{3}, 0.167\right),\left(p_{4}, 0.114\right),\left(p_{5}, 0.149\right)\right\}$.

Step 7: by using the optimal decision function $\max \left\{(f, \mathfrak{A})^{*}+(g, \mathfrak{B})^{*}(p)\right\}$, we have the ranking of the patients who are of high risk of COVID-19. The final ranking according to Algorithm 2 is given by

$$
p_{2}>p_{3}>p_{5}>p_{1}>p_{4}
$$

6.2. Comparison Analysis. Using NHS, cardinal sets, score matrices, and aggregate fuzzy sets, we produced two MAGDM techniques. Table 9 provides a comparison of both algorithms, showing the optimal alternative and results. Both algorithms provide the same optimum decision, as can be seen in the comparison table.

\section{Conclusion}

The idea of hypersoft sets is a newly emerging technique in dealing with problems in the real world. Herein, we have defined the new concept of semi-hypersoft sets of the fuzzy hypersoft topological space. Then, it has been extended to intuitionistic and neutrosophic semisets of intuitionistic and neutrosophic hypersoft topological spaces along with basic characterizations. Also, a real-life example in the current scenario of COVID-19 to make decision on the critical stage of medical diagnosis has been projected in MAGDM. This hypersoft topological space will also be extended to Pythagorean hypersoft topological spaces, as well as various forms of open sets, and more fuzzy topological space properties will be investigated. The concept of open sets introduced in this work may be extended to pre-, alpha-open neutrosophic hypersoft sets and strong open neutrosophic hypersoft sets based on which more such applications to real-world problems can be explored.

\section{Data Availability}

No data were used to support this study.

\section{Conflicts of Interest}

The authors declare that they have no conflicts of interest.

\section{Authors' Contributions}

N.B. contributed to funding acquisition. G.R. and N.B. conceptualized the study, contributed to software, performed formal analysis, developed the methodology, wrote the original draft, and validated the study. G.R., P.H., J.J.C., and D.A. supervised the study. G.R., D.A., and N.B. reviewed and edited the article. All authors read and agreed to the published version of the manuscript.

\section{Acknowledgments}

This research was supported by the Rajamangala University of Technology Suvarnabhumi.

\section{References}

[1] L. A. Zadeh, "Fuzzy sets," Information and Control, vol. 8, no. 3, pp. 338-353, 1965.

[2] K. T. Atanassov, "Intuitionistic fuzzy sets," Fuzzy Sets and Systems, vol. 20, no. 1, pp. 87-96, 1986.

[3] F. Smarandache, A unifying Field of Logics. Neutrosophy: Neutrosophic Probability, Set and Logic, American Research Press, Rehoboth, MA, USA, 1998.

[4] H. Wang, F. Smarandache, R. Sunderraman, and Y. Q. Zhang, "Single valued neutrosophic sets," Review of the Air Force Academy, vol. 4, pp. 410-413, 2010.

[5] J.-J. Peng, J.-Q. Wang, H.-Y. Zhang, and X.-H. Chen, "An outranking approach for multi-criteria decision-making problems with simplified neutrosophic sets," Applied Soft Computing, vol. 25, pp. 336-346, 2014.

[6] H. Zhang, J. Wang, and X. Chen, "An outranking approach for multi-criteria decision-making problems with intervalvalued neutrosophic sets," Neural Computing and Applications, vol. 27, no. 3, pp. 615-627, 2016.

[7] D. Karabasevi, D. Tanujkic, E. K. Zavadskas et al., "A novel extension of the TOPSIS method adapted for the use of singlevalued neutrosophic sets and hamming distance for E-commerce development strategies selection," Symmetry, vol. 12, p. $1263,2020$.

[8] D. Molodtsov, "Soft set theory-first results," Computers \& Mathematics with Applications, vol. 37, no. 4-5, pp. 19-31, 1999.

[9] P. K. Maji, R. Biswas, and A. R. Roy, "Soft set theory," Computers \& Mathematics with Applications, vol. 45, no. 4-5, pp. 555-562, 2003.

[10] F. Feng, Y. B. Jun, and X. Zhao, "Soft semirings," Computers \& Mathematics with Applications, vol. 56, no. 10, pp. 2621-2628, 2008.

[11] H. Aktap and N. Çağman, "Soft sets and soft groups," Information Sciences, vol. 177, no. 13, pp. 2726-2735, 2007.

[12] P. K. Maji, R. Biswas, and A. R. Roy, "Fuzzy soft sets," Journal of Fuzzy Mathematics, vol. 9, pp. 589-602, 2001.

[13] F. Smarandache, "Extension of soft set to hypersoft set, and then to plithogenic hypersoft set," Neutrosophic Sets and Systems, vol. 22, pp. 168-170, 2018. 
[14] M. Saqlain, M. Saeed, M. R. Ahmad, and F. Smarandache, "Generalization of TOPSIS for neutrosophic hypersoft set using accuracy function and its application," Neutrosophic Sets and Systems, vol. 27, pp. 131-137, 2019.

[15] F. Smarandache, "A Unifying Field in Logics, Neutrosophic Logic, Neutrosophy, Neutrosophic Set and Neutrosophic Probabilty, " Ed., American Research Press, Rehoboth, DE, USA, 4th edition, 1999.

[16] M. Abdel-Basset, A. Gamal, R. K. Chakrabortty, and M. Ryan, "A new hybrid multi-criteria decision-making approach for location selection of sustainable offshore wind energy stations: a case study," Journal of Cleaner Production, vol. 280, pp. 124-462, 2021.

[17] M. Abdel-Basset, G. Manogaran, and M. Mohamed, "A neutrosophic theory based security approach for fog and mobile-edge computing," Computer Networks, vol. 157, pp. 122-132, 2019.

[18] M. Abdel-Basst, R. Mohamed, and M. Elhoseny, "A model for the effective COVID-19 identification in uncertainty environment using primary symptoms and CT scans," Health Informatics Journal, vol. 26, no. 4, pp. 3088-3105, 2020.

[19] S. Rana, M. Qayyum, M. Saeed, F. Smarandache, and B. A. Khan, "Plithogenic fuzzy whole hypersoft set, construction of operators and their application in frequency matrix multi attribute decision making technique," Neutrosophic Sets and Systems, vol. 28, pp. 34-50, 2019.

[20] M. Saqlain, N. Jafar, S. Moin, M. Saeed, and S. Broumi, "Single and multi-valued neutrosophic hypersoft set and tangent similarity measure of single valued neutrosophic hypersoft sets," Neutrosophic Sets and Systems, vol. 32, pp. 317-329, 2020.

[21] M. Saqlain, S. Moin, M. N. Jafar, M. Saeed, and F. Smarandache, "Aggregate operators of neutrosophic hypersoft set," Neutrosophic Sets and Systems, vol. 32, pp. 294-306, 2020.

[22] M. Abbas, G. Murtaza, and F. Smarandache, "Basic operations on hypersoft sets and hypersoft point," Neutrosophic Sets and Systems, vol. 35, pp. 407-421, 2020.

[23] C. L. Chang, "Fuzzy topological spaces," Journal of Mathematical Analysis and Applications, vol. 24, no. 1, pp. 182-190, 1968.

[24] T. E. Gantner, R. C. Steinlage, and R. H. Warren, "Compactness in fuzzy topological spaces," Journal of Mathematical Analysis and Applications, vol. 62, no. 3, pp. 547-562, 1978.

[25] R. Lowen, "Convergence in fuzzy topological spaces," General Topology and Its Applications, vol. 10, no. 2, pp. 147-160, 1979.

[26] D. Çoker, "An introduction to intuitionistic fuzzy topological spaces," Fuzzy Sets and Systems, vol. 88, no. 1, pp. 81-89, 1997.

[27] A. A. Salama and S. A. Alblowi, "Neutrosophic set and neutrosophic topological spaces," IOSR Journal of Mathematics, vol. 3, no. 4, pp. 31-35, 2012.

[28] P. Iswarya and K. Bageerathi, "On neutrosophic semi-open sets in neutrosophic topological spaces," International Journal of Mathematics Trends and Technology, vol. 37, no. 3, pp. 214-223, 2016.

[29] B. Tanay and M. B. Kandemir, "Topological structure of fuzzy soft sets," Computers \& Mathematics with Applications, vol. 61, no. 10, pp. 2952-2957, 2011.

[30] A. Aygünoğlu and H. Aygün, "Some notes on soft topological spaces," Neural Computing and Applications, vol. 21, no. 1, pp. 113-119, 2012.

[31] S. Roy and T. K. Samanta, "An introduction to open and closed sets on fuzzy soft topological spaces," Annals of Fuzzy Mathematics and Informatics, vol. 6, no. 2, pp. 425-431, 2013.
[32] I. Osmanoglu and D. Tokat, "On intuitionistic Fuzzy soft topology," General Mathematics Notes, vol. 19, no. 2, pp. 59-70, 2013.

[33] S. Bayramov and C. Gunduz, "On intuitionistic fuzzy soft topological spaces," Turkic World Mathematical Society (TWMS) Journal of Pure and Applied Mathematics, vol. 5, no. 1, pp. 66-79, 2014.

[34] T. Bera and N. K. Mahapatra, "Introduction to neutrosophic soft topological space," Opsearch, vol. 54, no. 4, pp. 841-867, 2017.

[35] D. Ajay and J. J. Charisma, "Neutrosophic hypersoft topological spaces," Neutrosophic Sets and Systems, vol. 40, pp. 179-194, 2021.

[36] M. Riaz, B. Davvaz, A. Fakhar, and A. Firdous, "Hesitant fuzzy soft topology and its applications to multi-attribute group decision-making," Soft Computing, vol. 24, no. 21, pp. 16269-16289, 2020. 